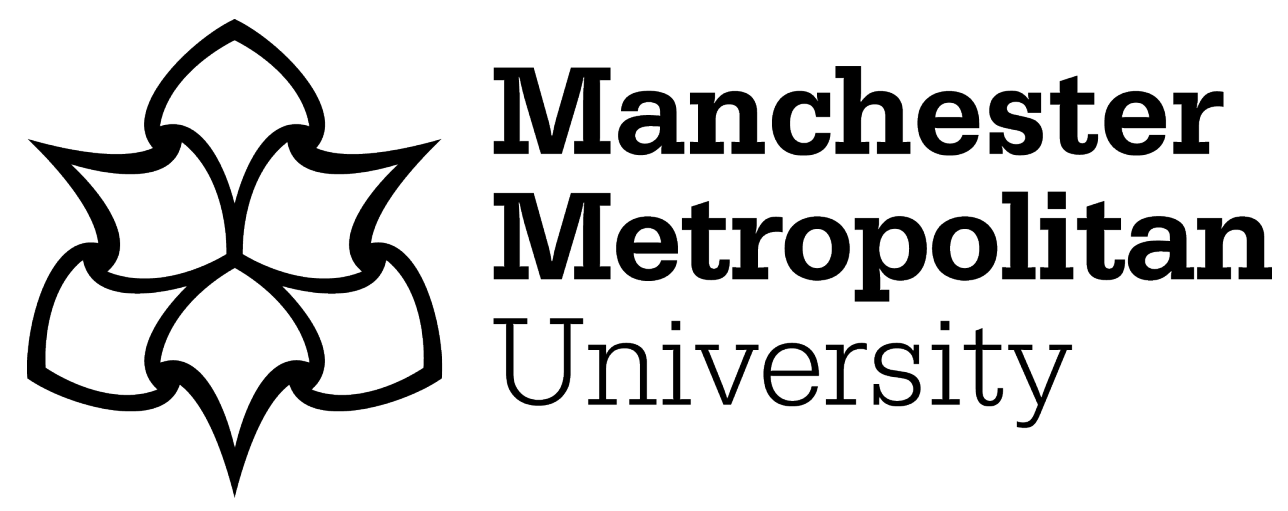

Pandey, Anshul, Yadav, Suneel, Do, Dinh-Thuan and Kharel, Rupak ORCID logoORCID: https://orcid.org/0000-0002-8632-7439 (2020) Secrecy Performance of Cooperative Cognitive AF Relaying Networks With Direct Links Over Mixed Rayleigh and Double-Rayleigh Fading Channels. IEEE Transactions on Vehicular Technology, 69 (12). pp. 15095-15112. ISSN 0018-9545

Downloaded from: https://e-space.mmu.ac.uk/627199/

Version: Accepted Version

Publisher: Institute of Electrical and Electronics Engineers (IEEE)

DOI: https://doi.org/10.1109/tvt.2020.3034729

Please cite the published version 


\title{
Secrecy Performance of Cooperative Cognitive AF Relaying Networks with Direct Links Over Mixed Rayleigh and Double-Rayleigh Fading Channels
}

\author{
Anshul Pandey, Student Member, IEEE, Suneel Yadav, Member, IEEE, Dinh-Thuan Do, Senior Member, IEEE, \\ and Rupak Kharel, Senior Member, IEEE
}

\begin{abstract}
This paper investigates the secrecy performance of an underlay cooperative cognitive relaying network, wherein a secondary source vehicle communicates with a fixed secondary destination terminal via a direct link and with the assistance of a secondary amplify-and-forward relay vehicle in the presence of a passive secondary eavesdropper vehicle, taking into consideration of interference at the primary user. We assume that the eavesdropper vehicle takes the advantages of both the relay link and direct link. We consider that vehicle-to-vehicle links are modeled as double-Rayleigh fading, while vehicle-tofixed infrastructure links are modeled as Rayleigh fading. Such a scenario finds it relevancy in vehicle-to-vehicle communication and/or vehicle-to-infrastructure communication under spectrum sharing heterogeneous cooperative vehicular networks. For such a realistic scenario, in particular, we derive a tight lower bound expression of the secrecy outage probability under mixed Rayleigh and double-Rayleigh fading channels. We also present an effective secrecy diversity order analysis and show that the considered system can achieve a secrecy diversity order of 2 for infinitely large average channel gain values of the main links. Finally, we demonstrate the accuracy of our analytical findings via numerical and simulation results and show the impact of channel conditions, primary interference constraints, and direct links on the secrecy performance of the considered system.
\end{abstract}

Index Terms-Physical-layer security, cooperative cognitive relaying networks, vehicle-to-vehicle communications, secrecy outage probability, double-Rayleigh fading channels.

\section{INTRODUCTION}

The evolution of $5 \mathrm{G}$ and beyond communication networks promises reliable connectivity and seamless integration of various complex heterogeneous networks to provide a truly digital world. One such key paradigm of $5 \mathrm{G}$ framework is driven by the advancements in the development of autonomous connected vehicles with embedded sensors forming vehicular communication networks [1]. These networks promise a plethora of mobility world applications, e.g., intelligent transportation system (ITS), environment monitoring, infotainment

Copyright (c) 2015 IEEE. Personal use of this material is permitted. However, permission to use this material for any other purposes must be obtained from the IEEE by sending a request to pubs-permissions@ieee.org.

A. Pandey and S. Yadav are with the Department of Electronics and Communication Engineering, Indian Institute of Information Technology Allahabad, India 211015 (e-mail: rse2016503@iiita.ac.in and suneel@iiita.ac.in).

D.-Thuan Do is with the Wireless Communications Research Group, Faculty of Electrical \& Electronics Engineering, Ton Duc Thang University, Ho Chi Minh 700000, Vietnam (Corresponding author, e-mail: dodinhthuan@tdtu.edu.vn).

R. Kharel is with the School of Engineering, Manchester Metropolitan University, Manchester M1 5GD, U.K. (e-mail: r.kharel@mmu.ac.uk). services etc. With such wide and real-time network access, a huge surge in the wireless data traffic is expected over the limited spectrum allocated for such networks apart from the challenges of maintaining a reliable connectivity, and information security and privacy. To this end, cooperative cognitive relaying technology can be used to support the shared spectrum access, and a better coverage and reliability for vehicle-toeverything (V2X) communications [2]-[4]. Furthermore, the aspects of cooperativeness, mobility, heterogeneity, dynamic nature of cognitive radios, and wireless transmissions have thrown up various new information security challenges in such networks [5]-[7]. Existing security solutions exploit key-based cryptographic techniques implemented on the upper layers, for which establishing an infrastructure may be difficult [7], [8].

Recently, physical-layer security (PHY-security) techniques have gained a wide research interest, as they exploit inherent nature of the wireless channels such as, fading, interference, to realize key-less secure transmissions via various coding and signal processing methods [8], [9]. Such techniques operate independently of the existing key-based security infrastructure and are efficient in terms of cost and resource utilization. Therefore, exploiting PHY-security in V2X enabled cooperative cognitive relay networks is the main focus of this paper.

\section{A. Related Works}

Owing to PHY-security advantages, the secrecy performance of cooperative relaying networks without adopting a cognitive spectrum sharing framework under various fading channels has been well examined in the literature (see [10]-[17] and the references therein). Specifically, the secrecy outage performance has been analyzed over Rayleigh fading in [10], [11], Nakagami- $m$ fading in [12]-[14], dual-correlated Rayleigh fading in [15], Rayleigh-dual correlated Rician fading in [16], and mixture Gamma distributed fading [17]. Of particular interest are the PHY-security in cooperative cognitive relaying networks, which has been studied thoroughly in the literature [18]-[33]. The authors in [18] and [19] investigated PHYsecurity performance of cooperative cognitive relay networks under Rayleigh fading. Furthermore, the authors in [20][23] studied the impact of relay diversity on the secrecy performance of such networks under Rayleigh fading. The authors in [24], [25] investigated PHY-security performance of the cooperative cognitive networks with multiple amplify-andforward (AF) relays in the presence of multiple eavesdroppers 
TABLE I

COMPARISON OF PROPOSED SySTEM WITH RELATED WORKS

\begin{tabular}{|c|c|c|c|c|c|c|}
\hline Context & This Work & [37], [38] & [39] & [40] & {$[41],[42]$} & [44] \\
\hline $\begin{array}{l}\text { Cooperative Cognitive } \\
\text { Relay Network }\end{array}$ & Yes & No & No & No & Yes & Yes \\
\hline Relaying Protocol & $\mathrm{AF}$ & $\mathrm{AF}$ & DF & $\mathrm{AF}$ & DF & $\mathrm{AF}$ \\
\hline Impact of Direct Links & Yes & Yes & No & No & No & No \\
\hline V2X Scenario & Yes & Yes & Yes & Yes & Yes & Yes \\
\hline PHY-Security & Yes & Yes & Yes & Yes & No & Yes \\
\hline Fading Scenario & $\begin{array}{l}\text { Mixed Rayleigh and } \\
\text { Double-Rayleigh }\end{array}$ & $\begin{array}{l}\text { Mixed Rayleigh and } \\
\text { Double-Rayleigh }\end{array}$ & Double-Rayleigh & $\begin{array}{l}\text { Mixed Nakagami- } m \text { and } \\
\text { Double Nakagami- } m\end{array}$ & $\begin{array}{l}\text { Mixed Rayleigh and } \\
\text { Double-Rayleigh }\end{array}$ & $\begin{array}{l}\text { Mixed Rayleigh and } \\
\text { Double-Rayleigh }\end{array}$ \\
\hline
\end{tabular}

over Rayleigh fading. The authors in [26] and [27] analyzed the secrecy performance of such networks with single and multiple relays, respectively, under Nakagami- $m$ fading. Recently, the residual energy maximization problem with multiple eavesdroppers has been studied over Rayleigh fading in [28]. The authors in [29] used relay selection alongwith jamming strategy to improve the network secrecy performance. The authors in [30] investigated PHY-security performance in cognitive Internet of Things (IoT) networks and proposed a jammer selection strategy. Moreover, the authors in [31] proposed an opportunistic source and jammer selection strategy among the multiple source nodes to improve the security of a cooperative cognitive network with multi-antenna relay and base-station in the presence of multi-antenna multiple eavesdroppers under Nakagami- $m$ fading. The authors in [32], [33] studied PHYsecurity with outdated channel estimates for such networks. However, the above studies for cooperative relay networks without cognitive framework [10]-[17] and with cognitive scenario [18]-[33] were confined to the environment, where the terminals are fixed and the channel between them is modeled as Rayleigh/Nakagami- $m$ /Rician fading.

On the other hand, Internet of Vehicles (IoV) and V2X communications are integral parts of the evolving $5 \mathrm{G}$ and beyond communication networks, which would be characterized by heterogeneity both in their link characteristics and embedded sensors computational ability. Specifically, such networks involve both vehicle-to-fixed infrastructure (V2I) and vehicle-to-vehicle $(\mathrm{V} 2 \mathrm{~V})$ links. Owing to the high mobility of vehicles, the classical Rayleigh or Nakagami- $m$ fading channels do not fit well for V2V communications [34]-[36]. Instead, cascaded Rayleigh (double-Rayleigh) channel modeling is shown to be more appropriate in characterizing the $\mathrm{V} 2 \mathrm{~V}$ communication links based on both the field measurement and theoretical analyses [34]-[36]. The secrecy performance analyses of cooperative vehicular networks over cascaded fading channels have been studied in [37]-[40]. However, all these works were carried out over non-cognitive framework. Albeit, the performance analysis of cooperative cognitive relay networks with cascaded fading channel modeling for V2V links is limited. The authors in [41] evaluated the performance of a cooperative cognitive network with multiple decode-andforward relays under double-Rayleigh fading. Also, the authors in [42] evaluated the performance of multi-hop cognitive radio networks with imperfect channel estimates over doubleRayleigh fading. However, the works [41] and [42] did not consider PHY-security aspects. Recently, the authors in [43] studied the trade-off between security and reliability for multi- hop cognitive V2V networks over double-Rayleigh fading. The authors in [44] evaluated PHY-security of cooperative cognitive vehicular relaying networks over cascaded fading.

\section{B. Motivation}

From the aforesaid works, it is evident that the authors in [37]-[40] investigated the secrecy performance of V2X enabled cooperative relay networks without considering the cognitive framework. However, the increasing demand of V2X applications and services have burdened the dedicated spectrum band allocated for such networks. The use of cognitive radio technology in $\mathrm{V} 2 \mathrm{X}$ enabled cooperative relay networks allows them to opportunistically access an additional spectrum band assigned for primary users without affecting the primary transmission. This introduces additional channels associated with the interfering links between secondary transmitters and primary receiver and brings the dependency in the channel gains of the instantaneous end-to-end signal-to-noise ratios at the destination and eavesdropper, which further makes the performance analysis of such V2X enabled cooperative cognitive relay networks more challenging and fundamentally different from [37]-[40]. Moreover, the efforts to study the performance of such networks without taking PHY-security into account have been made in [41], [42]. A little effort has been directed to analyze PHY-security in such networks with secondary V2V links [44], but the authors ignored the advantages of direct links (i.e., the channel between the sourceto-destination and between the source-to-eavesdropper). In practice, a direct link between transmitter and receiver can be available when the receiver does not experience severe fading or shadowing. Also, the direct links can affect both legitimate and wiretap transmissions, hence the impact of direct links should be considered for secure transmission in such networks.

To the best of authors' knowledge, there is no existing work which studied PHY-security in cooperative cognitive $\mathrm{AF}$ relay networks by exploiting the direct links under $\mathrm{V} 2 \mathrm{X}$ scenario, wherein V2V and V2I channels are modeled as double-Rayleigh fading and Rayleigh fading, respectively. This is because of the reason that the involvement of direct links in such networks under mixed fading environment makes the analysis more complex and intractable. Therefore, it is very important to investigate PHY-security in cooperative cognitive AF relay networks with direct links under mixed Rayleigh and double-Rayleigh fading channels. Further, Table I presents the comparison between the proposed work and existing works to clearly highlight the contributions of this paper. 


\section{Contributions}

This paper develops an understanding on PHY-security performance in $\mathrm{V} 2 \mathrm{X}$ enabled cooperative cognitive AF relay networks under mixed Rayleigh and double-Rayleigh fading. The theoretical findings of this paper thus 1) pose many mathematical challenges and complications due to the involved direct links and double-Rayleigh fading, 2) are unique as they are reported first time in the literature to examine PHYsecurity in the considered network with direct links and over mixed Rayleigh and double-Rayleigh fading, and 3) lay the foundation for analyzing PHY-security over more generalized cascaded fading models, such as, double Nakagami- $m$, $N^{*}$ Rayleigh, $N^{*}$ Nakagami-m. Particularly, in this paper, a secondary source $S$ communicates with a secondary receiver $D$ via a secondary AF relay $R$ and a direct link under the interference constraints of primary user $P U$. In addition, a secondary eavesdropper $E$ overhears the information from both $S$ and $R$. We assume that $S, R$, and $E$ are moving vehicles, whereas $P U$ and $D$ are stationary vehicles. By modeling V2V channels as double-Rayleigh fading and V2I channels as Rayleigh fading, we analyze system's secrecy performance. The key contributions of this paper are summarized as follows.

1) By considering V2V links ( $S \rightarrow R, S \rightarrow E$, and $R \rightarrow$ $E)$ as double-Rayleigh distributed and V2I links $(S \rightarrow$ $D, S \rightarrow P U, R \rightarrow P U$, and $R \rightarrow D$ ) as Rayleigh distributed, we derive the tight lower bound expressions for the cumulative distribution functions (CDFs) of the signal-to-noise ratios (SNRs) conditioned on the channel gains associated with $S \rightarrow P U$ and $R \rightarrow P U$ links.

2) Using the derived conditional CDFs, we deduce a tight lower bound secrecy outage probability (SOP) expression of the considered system under mixed Rayleigh and double-Rayleigh fading. Although the SOP expression involves Gauss-Laguerre series and infinite series, they converge to accurate values after a few terms.

3) By evaluating the asymptotic SOP behavior when average channel power gains of the main channels tend to infinity, we examine the effective secrecy diversity order and the secrecy array gain of the considered system. We demonstrate that the system can achieve a diversity order of 2, which is independent of the parameters related to eavesdropper and primary user. In addition, we also show the convergence behavior of secrecy diversity order under mixed Rayleigh and double-Rayleigh fading channels for different involved channel parameters.

4) We verify our analytical findings via numerical and simulation results, and show the impacts of channel conditions associated with $E$ and $P U$, and interference constraints on the SOP performance. Further, we illustrate that $S \rightarrow D$ and $S \rightarrow E$ direct links have significant effects on the system's SOP performance.

\section{Structure}

The remainder of this paper is organized as follows. Section II presents our system setup. In Section III, we derive the expression for the SOP, and analyze the effective secrecy diver-

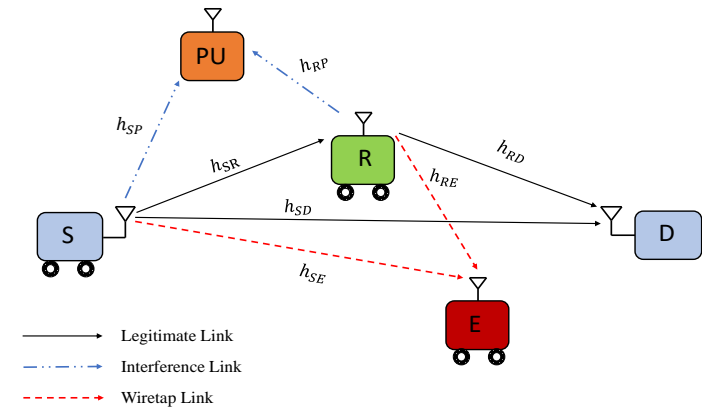

Fig. 1. The considered system model.

sity order. Section IV discusses the numerical and simulation results, and finally, the conclusions are drawn in Section V.

Notations: $\mathcal{K}_{v}(\cdot)$ denotes the $v$-th order modified Bessel function of second kind [45, eq. (8.432)], $\Psi(\cdot, \cdot, \cdot)$ being the Kummer hypergeometric function [45, eq. (9.210)], $G_{p, q}^{m, n}\left(\left.x\right|_{b_{1}, \cdots, b_{q}} ^{a_{1}, \cdots, a_{p}}\right)$ is the Meijer- $G$ function [45, eq. (9.301)], $G_{p_{1}, q_{1}: p_{2}, q_{2}: p_{3}, q_{3}}^{m_{1}, n_{1}: n_{2}, m_{2}: n_{3}, m_{3}}\left(x,\left.\left.\left.y\right|_{b_{1}, \cdots, b_{q_{1}}} ^{a_{1}, \cdots, a_{p_{1}}}\right|_{d_{1}, \cdots, d_{q_{2}}} ^{c_{1}, \cdots, c_{p_{2}}}\right|_{f_{1}, \cdots, f_{q_{3}}} ^{e_{1}, \cdots, e_{p_{3}}}\right)$ is the extended generalized bi-variate Meijer- $G$ function [46, eq. (07.34.21.0081.01)], and $\mathcal{W}_{u, v}(z)$ denotes the Whittaker- $\mathcal{W}$ function [46, eq. (07.45.02.0001.01)].

\section{System And CHANNEL Models}

We consider a secure cooperative cognitive relay network as depicted in Fig. 1, where the nodes in the secondary network communicate over the same licensed spectral band allocated for the primary network in underlay mode. Such systems can find their practical relevancy in many futuristic communication systems, such as, cognitive IoT networks [30], cognitive radio sensor networks [42], and cognitive vehicular relaying networks [47], [48]. In particular, the primary network consists of a $P U$, whereas the secondary network comprises of $S, R$, and $D$ nodes. An $E$ also exists in the network to eavesdrop the information. Each node has a single-antenna and operate in half-duplex manner. Under passive eavesdropping scenario, the instantaneous channel state information (CSI) between $S$ and $E(R$ and $E$ ) is not available at $S(R)$. Note that in practice the eavesdropper is generally not cooperative and not willing to feedback its instantaneous CSI to the legitimate system [10]. Since the information of eavesdropper channel does not change under the consideration of quasi-stationary channel for a short duration (i.e., one fading block time), therefore only statistical CSI of the eavesdropper is available at the legitimate nodes. Also, during the overall process $S$ and $R$ impose the interference at $P U$, since there is no detection strategy available at $P U$ to detect the interference impinged by $S$ and $R$. Moreover, we assume all the channels to be quasi-static, reciprocal, and subject to independent and non-identically distributed fading. Further, V2V channels, i.e., $S \rightarrow R, S \rightarrow E$, and $R \rightarrow E$ are assumed to experience double-Rayleigh fading. In particular, the channel coefficients $h_{\imath \jmath}$ for $\{\imath \jmath\} \in\{S R, S E, R E\}$ can be modeled as the product of $h_{\imath \jmath, 1}$ and $h_{\imath \jmath, 2}$, where $h_{\imath \jmath, 1}$ and $h_{\imath \jmath, 2}$ are independent 
complex Gaussian random variables having zero mean and variance $\Omega_{\imath \jmath, 1}$ and $\Omega_{\imath \jmath, 2}$, respectively. On the other side, $h_{S P}$, $h_{R P}, h_{R D}$, and $h_{S D}$ are the channel coefficients for $S \rightarrow P U$, $R \rightarrow P U, R \rightarrow D$, and $S \rightarrow D$ V2I links, respectively, which can be modeled as $\mathcal{C N}\left(0, \Omega_{S P}\right), \mathcal{C N}\left(0, \Omega_{R P}\right), \mathcal{C N}\left(0, \Omega_{R D}\right)$, and $\mathcal{C N}\left(0, \Omega_{S D}\right)$, respectively. The additive white Gaussian noise (AWGN) for each link is modeled as $\mathcal{C N}\left(0, N_{0}\right)$.

In particular, $S$ requires two transmission phases to transmit secret information to $D$. During the first transmission phase, $S$ broadcasts a unit energy signal $x_{s}$ with power $P_{S}$, and hence the received signal at $R$ and receiver $i$, for $i \in\{D, E\}$, can be given as $y_{R}=\sqrt{P_{S}} h_{S R} x_{s}+n_{R}, y_{i}^{(1)}=\sqrt{P_{S}} h_{R i} x_{s}+$ $n_{i}^{(1)}$, respectively, where $n_{R}$ and $n_{i}^{(1)}$ denote the AWGNs at the receivers $R$ and $i$ in the first transmission phase. In the second transmission phase, $R$ amplifies the received signal $y_{R}$ with a variable gain $\mathcal{A}=\sqrt{\frac{P_{R}}{P_{S}\left|h_{S R}\right|^{2}+N_{0}}}$, and broadcasts the resultant signal to $D$, where $P_{R}$ is the relay transmit power. Moreover, $E$ in the network overhears the relayed information. Therefore, the signal received at receiver $i$, for $i \in\{D, E\}$, can be given by $y_{i}^{(2)}=\mathcal{A} h_{R i} y_{R}+n_{i}^{(2)}$, where $n_{i}^{(2)}$ denotes the AWGN at receiver $i$ in the second transmission phase. Consequently, the instantaneous end-to-end SNRs received at receiver $i$ via relay link (i.e., $S \rightarrow R \rightarrow i$ ) and direct link (i.e., $S \rightarrow i$ ), for $i \in\{D, E\}$, can be given, respectively, by

$$
\begin{aligned}
& \Lambda_{S \rightarrow R \rightarrow i}=\frac{P_{S} P_{R}\left|h_{S R}\right|^{2}\left|h_{R i}\right|^{2}}{P_{S}\left|h_{S R}\right|^{2} N_{0}+P_{R}\left|h_{R i}\right|^{2} N_{0}+N_{0}^{2}}, \\
& \Lambda_{S \rightarrow i}=\frac{P_{S}\left|h_{S i}\right|^{2}}{N_{0}} .
\end{aligned}
$$

Moreover, by applying the maximum ratio combining scheme, the resultant SNR at receiver $i$, for $i \in\{D, E\}$, using (1) and (2) can be given by $\digamma_{i}=\Lambda_{S \rightarrow i}+\Lambda_{S \rightarrow R \rightarrow i}$.

According to underlay cognitive radio transmission, $P_{S}$ and $P_{R}$ must be limited as to keep the interference imposed on $P U$ below the maximum tolerable interference level $Q$ [19]-[21]. Therefore, the transmit powers at $S$ and $R$ are constrained ${ }^{1}$ by $P_{S}=\frac{\mathcal{Q}}{\left|h_{S P}\right|^{2}}$ and $P_{R}=\frac{\mathcal{Q}}{\left|h_{R P}\right|^{2}}$, respectively, for ensuring the QoS of $P U$. Using these constraints via (1) and (2), we can obtain the resultant SNR at receiver $i$, for $i \in\{D, E\}$, as

$$
\digamma_{i}=\Lambda_{S i}+\Lambda_{i}
$$

where $\Lambda_{i} \triangleq \frac{\Lambda_{S R} \Lambda_{R i}}{\Lambda_{S R}+\Lambda_{R i}+1}, \Lambda_{S R}=\frac{\rho\left|h_{S R}\right|^{2}}{\left|h_{S P}\right|^{2}}, \Lambda_{R i}=\frac{\rho\left|h_{R i}\right|^{2}}{\left|h_{R P}\right|^{2}}$, $\Lambda_{S i}=\frac{\rho\left|h_{S i}\right|^{2}}{\left|h_{S P}\right|^{2}}$, for $i \in\{D, E\}$, and $\rho=\frac{\mathcal{Q}}{N_{0}}$ as the transmit $\mathrm{SNR}$. Moreover, the capacity pertaining to $S \rightarrow R \rightarrow i$ link can be defined as $\mathcal{C}_{i}=\frac{1}{2} \log _{2}\left(1+\digamma_{i}\right)$. Thus, the system secrecy capacity is given by $\mathcal{C}_{\mathrm{sec}}=\max \left(\mathcal{C}_{D}-\mathcal{C}_{E}, 0\right)$.

It should be noted that under double-Rayleigh fading, the CDF and the probability distribution function (PDF) for the channel gain $\left|h_{\imath \jmath}\right|^{2}$ (assuming $\Omega_{\imath \jmath, 1}=\Omega_{\imath \jmath, 2}=\Omega_{\imath \jmath}$ ),

\footnotetext{
${ }^{1}$ Here, we consider that the secondary vehicles share the licensed spectrum to guarantee the quality-of-service (QoS) of $P U$, without taking into account the specific QoS requirements of vehicular applications. However, there exist several scenarios where the spectrum allocation is performed to meet the QoS requirements of vehicular applications, such as bandwidth usage for non-safety applications and delay constraints for safety-related applications [48], [49]. Such scenarios may involve some additional QoS requirement constraints/parameters, whose study is beyond the scope of this work.
}

for $\{\imath \jmath\} \in\{S R, S E, R E\}$, are given as $F_{\left|h_{\imath \jmath}\right|^{2}}(y)=$ $1-\left(2 / \Omega_{\imath \jmath}\right) \sqrt{y} \mathcal{K}_{1}\left(\left(2 / \Omega_{\imath \jmath}\right) \sqrt{y}\right), y>0$ and $f_{\left|h_{\imath \jmath}\right|^{2}}(y)=$ $\left(2 / \Omega_{\imath \jmath}^{2}\right) \mathcal{K}_{0}\left(\left(2 / \Omega_{\imath \jmath}\right) \sqrt{y}\right), y>0$, respectively. Moreover, under Rayleigh fading, the CDF and PDF of $\left|h_{a b}\right|^{2}$, for $\{a b\} \in$ $\{S D, S P, R P, R D\}$, can be expressed as $F_{\left|h_{a b}\right|^{2}}(y)=1-$ $\mathrm{e}^{-\frac{y}{\Omega_{a b}}}, y>0$ and $f_{\left|h_{a b}\right|^{2}}(y)=\frac{1}{\Omega_{a b}} \mathrm{e}^{-\frac{y}{\Omega_{a b}}}, y>0$, respectively.

\section{Performance Analysis}

This section investigates the system's secrecy performance in terms of SOP and effective secrecy diversity order. Before proceeding further, we first derive the tight lower bound of the CDFs of $\digamma_{D}$ and $\digamma_{E}$ conditioned on $\left|h_{S P}\right|^{2}$ and $\left|h_{R P}\right|^{2}$ (as $\digamma_{D}$ and $\digamma_{E}$ are dependent due to the involvement of common channel gains $\left|h_{S P}\right|^{2}$ and $\left|h_{R P}\right|^{2}$ ), in the sequel. This will help us to evaluate the performance measures under investigation.

\section{A. CDFs of $\digamma_{D}$ and $\digamma_{E}$ conditioned on $\left|h_{S P}\right|^{2}$ and $\left|h_{R P}\right|^{2}$}

Since $\digamma_{D}$ and $\digamma_{E}$ are dependent because of the involved common channel gains $X \triangleq\left|h_{S P}\right|^{2}$ and $Z \triangleq\left|h_{R P}\right|^{2}$, therefore, we first need to derive the conditional CDFs $F_{\digamma_{D}}(y \mid X, Z)$ and $F_{\digamma_{E}}(y \mid X, Z)$ using (3). To proceed, without loss of generality, we use the fact that the harmonic mean of two positive numbers can be upper bounded by the minimum of those two numbers, ${ }^{2}$ i.e., $\frac{x y}{x+y+1} \simeq \frac{x y}{x+y}<\min (x, y)$ [11], [37], and hence the upper bound of $\Lambda_{D}$ and $\Lambda_{E}$ can be given as $\widetilde{\Lambda}_{D}<\min \left(\Lambda_{S R}, \Lambda_{R D}\right)$ and $\widetilde{\Lambda}_{E}<\min \left(\Lambda_{S R}, \Lambda_{R E}\right)$, respectively. Thus, we can have $\digamma_{D}=\Lambda_{S D}+\widetilde{\Lambda}_{D}$ and $\digamma_{E}=\Lambda_{S E}+\widetilde{\Lambda}_{E}$ with $\widetilde{\Lambda}_{D}=\min \left(\Lambda_{S R}, \Lambda_{R D}\right)$ and $\widetilde{\Lambda}_{E}=$ $\min \left(\Lambda_{S R}, \Lambda_{R E}\right)$. Consequently, the lower bound (LB) for the CDFs $F_{\digamma_{i}}(y \mid X, Z)$, for $i \in\{D, E\}$, can be expressed as

$$
F_{\digamma_{i}}^{\mathrm{LB}}(y \mid X, Z)=\int_{0}^{x} F_{\widetilde{\Lambda}_{i}}(y-x \mid X, Z) f_{\Lambda_{S i}}(x \mid X) d x .
$$

To evaluate (4), we first require the CDF of $\widetilde{\Lambda}_{i}$ conditioned on $X, Z$, for $i \in\{D, E\}$, as given in Proposition 1 .

Proposition 1: Using the CDFs of $\Lambda_{S R}, \Lambda_{R D}$, and $\Lambda_{R E}$, the conditional CDFs $F_{\widetilde{\Lambda}_{D}}(y \mid X, Z)$ and $F_{\widetilde{\Lambda}_{E}}(y \mid X, Z)$ are given by

$$
\begin{aligned}
F_{\widetilde{\Lambda}_{D}}(y \mid X, Z) & =1-2 \alpha_{S R} \mathrm{e}^{-\alpha_{R D} y Z} \sqrt{y X} \mathcal{K}_{1}\left(2 \alpha_{S R} \sqrt{y X}\right), \\
F_{\widetilde{\Lambda}_{E}}(y \mid X, Z) & =1-4 y \alpha_{S R} \alpha_{R E} \sqrt{X Z} \mathcal{K}_{1}\left(2 \alpha_{S R} \sqrt{y X}\right) \\
& \times \mathcal{K}_{1}\left(2 \alpha_{R E} \sqrt{y Z}\right)
\end{aligned}
$$

where $\alpha_{S R}=\frac{1}{\sqrt{\rho} \Omega_{S R}}, \alpha_{R E}=\frac{1}{\sqrt{\rho} \Omega_{R E}}$, and $\alpha_{R D}=\frac{1}{\rho \Omega_{R D}}$.

Proof: See Appendix A for the detailed derivation.

Remark 1: From Proposition 1, it is noted that i) the conditional CDFs in (5) and (6) mainly involve exponential function and/or modified Bessel functions of second kind, which can easily be evaluated for various values of involved parameters, and hence make them mathematically tractable to use, and ii) Proposition 1 will be useful in deriving the conditional CDFs of instantaneous SNRs, $\digamma_{i}^{\mathrm{LB}}$, for $i \in\{D, E\}$, in (4).

Remark 2: By invoking (5) and the conditional PDF $f_{\Lambda_{S D}}(y \mid X)$ (can be obtained by differentiating conditional

\footnotetext{
${ }^{2}$ Note that the bound is quite accurate if $x$ is far from $y$, while the accuracy decreases if $x$ is close to $y$. The bound becomes exact as $x$ and $y$ go to infinity. The tightness of this bound is shown in Table-II under the SOP analysis.
} 
TABLE II

SOP DifFERENCE BETWEen EXACT EXPRESSION AND APPROXIMATE EXPRESSION (WITH $\Omega_{S D}=0$ DB AND $\mathcal{R}_{s}=0.1 \mathrm{BPS} / \mathrm{Hz}$ )

\begin{tabular}{|c|c|c|c|c|c|c|c|c|c|c|c|}
\hline SNR (dB) & 0 & 5 & 10 & 15 & 20 & 25 & 30 & 35 & 40 & 45 & 50 \\
\hline $\begin{array}{l}\text { Exact }\left(\Omega_{S R}=\Omega_{R D}=0 \mathrm{~dB}, \Omega_{S E}=\Omega_{R E}=\right. \\
\left.0 \mathrm{~dB}, \Omega_{S P}=\Omega_{R P}=0 \mathrm{~dB}\right)\end{array}$ & 0.4998 & 0.4595 & 0.4426 & 0.4373 & 0.4372 & 0.4334 & 0.4368 & 0.4345 & 0.4374 & 0.4338 & 0.4397 \\
\hline $\begin{array}{l}\text { Approximate }\left(\Omega_{S R}=\Omega_{R D}=0 \mathrm{~dB}, \Omega_{S E}=\right. \\
\left.\Omega_{R E}=0 \mathrm{~dB}, \Omega_{S P}=\Omega_{R P}=0 \mathrm{~dB}\right)\end{array}$ & 0.4978 & 0.4573 & 0.4449 & 0.4393 & 0.4397 & 0.4360 & 0.4349 & 0.4366 & 0.4371 & 0.4360 & 0.4370 \\
\hline Difference in percentage & $0.4 \%$ & $0.48 \%$ & $0.51 \%$ & $0.45 \%$ & $0.56 \%$ & $0.59 \%$ & $0.43 \%$ & $0.48 \%$ & $0.06 \%$ & $0.5 \%$ & $0.61 \%$ \\
\hline $\begin{array}{l}\text { Exact }\left(\Omega_{S R}=10 \mathrm{~dB}, \Omega_{R D}=0 \mathrm{~dB}, \Omega_{S E}=\right. \\
\left.\Omega_{R E}=0 \mathrm{~dB}, \Omega_{S P}=\Omega_{R P}=0 \mathrm{~dB}\right)\end{array}$ & 0.4886 & 0.4658 & 0.4591 & 0.4532 & 0.4506 & 0.4545 & 0.4535 & 0.4532 & 0.4507 & 0.4519 & 0.4514 \\
\hline $\begin{array}{l}\text { Approximate }\left(\Omega_{S R}=10 \mathrm{~dB}, \Omega_{R D}=0 \mathrm{~dB},\right. \\
\left.\Omega_{S E}=\Omega_{R E}=0 \mathrm{~dB}, \Omega_{S P}=\Omega_{R P}=0 \mathrm{~dB}\right)\end{array}$ & 0.4880 & 0.4644 & 0.4565 & 0.4547 & 0.4529 & 0.4535 & 0.4511 & 0.4521 & 0.4510 & 0.4538 & 0.4534 \\
\hline Difference in percentage & $0.12 \%$ & $0.3 \%$ & $0.56 \%$ & $0.32 \%$ & $0.5 \%$ & $0.22 \%$ & $0.53 \%$ & $0.24 \%$ & $0.06 \%$ & $0.41 \%$ & $0.44 \%$ \\
\hline $\begin{array}{l}\text { Exact }\left(\Omega_{S R}=\Omega_{R D}=10 \mathrm{~dB}, \Omega_{S E}=\right. \\
\left.\Omega_{R E}=10 \mathrm{~dB}, \Omega_{S P}=\Omega_{R P}=10 \mathrm{~dB}\right)\end{array}$ & 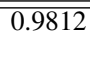 & 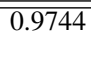 & 0.9734 & 0.9728 & 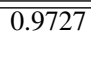 & 0.9724 & 0.9708 & 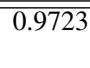 & 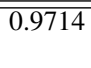 & 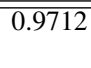 & 0.9725 \\
\hline $\begin{array}{l}\text { Approximate }\left(\Omega_{S R}=\Omega_{R D}=10 \mathrm{~dB}, \Omega_{S E}=\right. \\
\left.\Omega_{R E}=10 \mathrm{~dB}, \Omega_{S P}=\Omega_{R P}=10 \mathrm{~dB}\right)\end{array}$ & 0.9753 & 0.9691 & 0.9668 & 0.9678 & 0.9660 & 0.9671 & 0.9655 & 0.9666 & 0.9675 & 0.9659 & 0.9662 \\
\hline Difference in percentage & $0.6 \%$ & $0.54 \%$ & $0.68 \%$ & $0.51 \%$ & $0.69 \%$ & $0.54 \%$ & $0.54 \%$ & $0.58 \%$ & $0.4 \%$ & $0.54 \%$ & $0.65 \%$ \\
\hline
\end{tabular}

CDF $F_{\Lambda_{S D}}(y \mid X)=1-\mathrm{e}^{-\alpha_{S D} y X}$ with respect to $y$, where $\alpha_{S D}=\frac{1}{\rho \Omega_{S D}}$ ) into (4), it is observed that the integral involved in evaluating $F_{\digamma_{D}}^{\mathrm{LB}}(y \mid X, Z)$ comprises of an exponential term, an algebraic term, and a shifted version of modified Bessel function of second kind (e.g., $\mathcal{K}_{v}(\sqrt{y-x})$, whose closed-form solution is tedious and intractable. Also, substituting (6) and the conditional PDF $f_{\Lambda_{S E}}(y \mid X)$ (can be calculated by differentiating conditional $\mathrm{CDF} F_{\Lambda_{S E}}(y \mid X)=1-2 \alpha_{S E} \sqrt{y X} \mathcal{K}_{1}\left(2 \alpha_{S E} \sqrt{y X}\right)$ with respect to $y$, where $\alpha_{S E}=\frac{1}{\sqrt{\rho} \Omega_{S E}}$ ) into (4), we need to simplify an integral that involves powers and the product of three Bessel functions of different orders and arguments (e.g., $\left.\mathcal{K}_{u}(\sqrt{y}) \mathcal{K}_{v}(a \sqrt{y-x}) \mathcal{K}_{v}(b \sqrt{y-x})\right)$, whose solution does not exist. This implies that the exact evaluation of (4) using (5) and (6) seems mathematically intractable.

Remark 3: The effective and tractable evaluation of (4) can be obtained by applying a tight staircase approximation [51] to the original triangular integral region in $\digamma_{D}$ and $\digamma_{E}$ with $M$ tractable rectangular sub-regions to evaluate $F_{\digamma_{D}}^{\mathrm{LB}}(y \mid X, Z)$ and $F_{\digamma_{E}}^{\mathrm{LB}}(y \mid X, Z)$, as given in Proposition 2.

Proposition 2: The conditional CDFs $F_{\digamma_{D}}^{\mathrm{LB}}(y \mid X, Z)$ and $F_{\digamma_{E}}^{\mathrm{LB}}(y \mid X, Z)$ in (4) can be expressed as

$F_{\digamma_{D}}^{\mathrm{LB}}(y \mid X, Z)=\sum_{k=1}^{M}\left[\mathrm{e}^{-\alpha_{S D} X \tau_{k 1} y}-2 \alpha_{S R} \sqrt{X} \mathrm{e}^{-\alpha_{S D} X \tau_{k 1} y}\right.$

$\times \mathrm{e}^{-\left(1-\tau_{k 1}\right) \alpha_{R D} Z y} \sqrt{y} \mathcal{K}_{1}\left(2 \alpha_{S R} \sqrt{y X}\right)-\mathrm{e}^{-\alpha_{S D} X \tau_{k} y}+2 \alpha_{S R}$

$\left.\times \sqrt{X} \mathrm{e}^{\left.-\left[\alpha_{S D} X \tau_{k}+\left(1-\tau_{k 1}\right) \alpha_{R D} Z\right)\right] y} \sqrt{y} \mathcal{K}_{1}\left(2 \alpha_{S R} \sqrt{y X}\right)\right]$,

$F_{\digamma_{E}}^{\mathrm{LB}}(y \mid X, Z)=\left(1-2 \alpha_{S E} \sqrt{\frac{y X}{M}} \mathcal{K}_{1}\left(2 \alpha_{S E} \sqrt{\frac{y X}{M}}\right)\right)$

$\times\left(1-4 y \alpha_{S R} \alpha_{R E} \sqrt{X Z} \mathcal{K}_{1}\left(2 \alpha_{S R} \sqrt{y X}\right) \mathcal{K}_{1}\left(2 \alpha_{R E} \sqrt{y Z}\right)\right)$

$+\sum_{j=2}^{M}\left[\left(2 \alpha_{S E} \sqrt{\tau_{j 1} X} \mathcal{K}_{1}\left(2 \alpha_{S E} \sqrt{\tau_{j 1} y X}\right)-2 \alpha_{S E} \sqrt{\tau_{j} y X}\right.\right.$

$\left.\times \mathcal{K}_{1}\left(2 \alpha_{S E} \sqrt{\tau_{j} y X}\right)\right)\left(1-4\left(1-\tau_{j 1}\right) y \alpha_{S R} \alpha_{R E} \sqrt{X Z}\right.$

$\left.\left.\times \mathcal{K}_{1}\left(2 \alpha_{S R} \sqrt{\left(1-\tau_{j 1}\right) y X}\right) \mathcal{K}_{1}\left(2 \alpha_{R E} \sqrt{\left(1-\tau_{j 1}\right) y Z}\right)\right)\right]$,

where $\tau_{k}=\frac{k}{M}, \tau_{k 1}=\frac{k-1}{M}, \tau_{j}=\frac{j}{M}$, and $\tau_{j 1}=\frac{j-1}{M}$.
Proof: See Appendix B for the proof.

Remark 4: From Proposition 2, it is to be emphasized that i) the conditional CDFs in (7) and (8) mainly consist of exponential function of type $\mathrm{e}^{-a y}$ and modified Bessel function of second kind of type $\mathcal{K}_{1}(b \sqrt{y}) . \mathcal{K}_{1}(b \sqrt{y})$ can be further expressed as $\mathcal{K}_{1}(b \sqrt{y})=2 b \sqrt{y} \sqrt{\pi} \mathrm{e}^{-b \sqrt{y}} \Psi(1.5,3 ; 2 b \sqrt{y})[45$, eq. (9.210)], and realizing this in (7) and (8) implies that Proposition 2 mainly depend on $\mathrm{e}^{-a y}$ and/or $\mathrm{e}^{-b \sqrt{y}}$, and decrease rapidly as $y$ increases; ii) Proposition 2 also depend on $M$ tractable rectangular sub-regions, whose value can be appropriately selected to demonstrate the tightness of the used approximation, as shown numerically under Section IV.

Remark 5: The derived conditional CDFs $F_{\digamma_{D}}^{\mathrm{LB}}(y \mid X, Z)$ and $F_{\digamma_{E}}^{\mathrm{LB}}(y \mid X, Z)$ in Proposition 2, to our best knowledge, are new and will be used to evaluate the SOP in Section III-B and the effective secrecy diversity order in Section III-C.

\section{B. Secrecy Outage Probability (SOP)}

The SOP informs us what is the probability that the instantaneous secrecy capacity falls below a predefined secrecy target rate $\mathcal{R}_{s}$ (in bps/Hz). Mathematically, it can be expressed as

$$
\mathcal{P}_{\text {out }}^{\text {sec }}=\operatorname{Pr}\left\{\max \left[\mathcal{C}_{D}-\mathcal{C}_{E}, 0\right]<\mathcal{R}_{s}\right\} .
$$

From (9), it is seen that when $\mathcal{C}_{D} \leq \mathcal{C}_{E}$, the system security is compromised i.e., $\mathcal{P}_{\text {out }}^{\text {sec }}=1$. Thus, we resort our SOP analysis for $\mathcal{C}_{\mathrm{D}}>\mathcal{C}_{\mathrm{E}}$, and consequently (9) can be expressed as $\mathcal{P}_{\text {out }}^{\mathrm{sec}}=$ $\operatorname{Pr}\left[\mathcal{C}_{D}-\mathcal{C}_{E}<\mathcal{R}_{s}\right]$. Thus, a lower bound ${ }^{3}$ of $\mathcal{P}_{\text {out }}^{\text {sec }}$ is given by

$$
\mathcal{P}_{\text {sec }}^{\text {out, }}{ }^{\mathrm{LB}}(\eta)=\operatorname{Pr}[\Theta<\eta],
$$

where $\Theta=\frac{1+\digamma_{D}}{1+\digamma_{E}}$ with $\digamma_{D}=\Lambda_{S D}+\widetilde{\Lambda}_{D}, \digamma_{E}=\Lambda_{S E}+\widetilde{\Lambda}_{E}$, $\widetilde{\Lambda}_{D}=\min \left(\Lambda_{S R}, \Lambda_{R D}\right), \widetilde{\Lambda}_{E}=\min \left(\Lambda_{S R}, \Lambda_{R E}\right)$, and $\eta=$ $2^{2 \mathcal{R}_{s}}$ denotes the secrecy target threshold.

${ }^{3}$ Note that a lower bound of SOP can be evaluated by applying an upper bound $\frac{x y}{x+y}<\min (x, y)$ [11], [37], as it provides tight results over a broad range of SNR $\left(\rho=\frac{\mathcal{Q}}{N_{0}}\right)$. The Monte-Carlo simulations presented in Table II (shown at the top of the page) demonstrate that the difference between the exact SOP expression (i.e., $\left.\operatorname{Pr}\left[\frac{1+\Lambda_{S D}+\frac{\Lambda_{S R} \Lambda_{R D}}{\Lambda_{S R}+\Lambda_{R D}+1}}{1+\Lambda_{S E}+\frac{\Lambda_{S R} \Lambda_{R E}}{\Lambda_{S R}+\Lambda_{R E}+1}}\right]\right)$ and the approximate SOP expression $\left(\right.$ i.e., $\left.\operatorname{Pr}\left[\frac{1+\Lambda_{S D}+\min \left(\Lambda_{S R}, \Lambda_{R D}\right)}{1+\Lambda_{S E}+\min \left(\Lambda_{S R}, \Lambda_{R E}\right)}\right]\right)$, for various set of involved parameters, is very small. 
TABLE III

AUXILIARY FUNCTIONS USED IN SOP EXPRESSION

\begin{tabular}{|c|c|}
\hline $\mathcal{C}_{1}(\theta, X)=\mathrm{e}^{-\varpi_{k 1}(\theta-1) X}$ & $\mathcal{N}_{1}\left(\kappa_{1}, \kappa_{2}\right)=G_{2,0: 0,1: 0,2}^{0,2: 1,0: 2,0}\left(\left.\left.\begin{array}{r}-1,0 \\
-\end{array}\right|_{0} ^{-}\right|_{0,0} ^{-} \mid \kappa_{1}, \kappa_{2}\right)$ \\
\hline $\mathcal{C}_{2}(\theta, X)=\mathrm{e}^{-\varpi_{k}(\theta-1) X}$ & $\mathcal{N}_{2}\left(\beta_{1}, \beta_{2}, \beta_{3}, \beta_{4}, \beta_{5}, \beta_{6}, \kappa_{1}, \kappa_{2}\right)=G_{2,0: 0,2: 0,2}^{0,2: 2,0: 2,0}\left(\left.\left.\stackrel{\beta_{1}, \beta_{2}}{-}\right|_{\beta_{3}, \beta_{4}} ^{-}\right|_{\beta_{5}, \beta_{6}} ^{-} \mid \kappa_{1} \frac{\alpha_{R E}^{2}}{\alpha_{S E}^{2}}, \kappa_{2} \frac{\alpha_{S R}^{2}}{\alpha_{S E}^{2}}\right)$ \\
\hline$\varpi_{k}=\alpha_{S D} \tau_{k}$ & $\zeta_{1}\left(y_{l_{1}} ; \theta, X\right)=2 \alpha_{S R} \sqrt{\left(\theta-1+\theta y_{l_{1}}\right) X} \mathcal{K}_{1}\left(2 \alpha_{S R} \sqrt{\left(\theta-1+\theta y_{l_{1}}\right) X}\right)$ \\
\hline$\varpi_{k 1}=\alpha_{S D} \tau_{k 1}$ & $\xi_{1}\left(y ; \kappa_{1}, \kappa_{2}, \kappa_{3}, a_{1}, a_{2}, b_{1}, b_{2}\right)=4 X^{\kappa_{1}} Z^{\kappa_{2}} y^{\kappa_{3}} \mathcal{K}_{a_{1}}\left(2 b_{1} \sqrt{y}\right) \mathcal{K}_{a_{2}}\left(2 b_{2} \sqrt{y}\right)$ \\
\hline $\begin{array}{l}\mu_{k 1}\left(y_{l_{1}} ; \theta, X, Z\right)=\left(\alpha_{S D} X \tau_{k 1}+\right. \\
\left.\left(1-\tau_{k 1}\right) \alpha_{R D} Z\right)\left(\theta-1+\theta y_{l_{1}}\right)\end{array}$ & $\xi_{2}\left(y ; \kappa_{1}, \kappa_{2}, \kappa_{3}, a_{1}, a_{2}, a_{3}, b_{1}, b_{2}, b_{3}\right)=8 X^{\kappa_{1}} Z^{\kappa_{2}} y^{\kappa_{3}} \mathcal{K}_{a_{1}}\left(2 b_{1} \sqrt{y}\right) \mathcal{K}_{a_{2}}\left(2 b_{2} \sqrt{y}\right) \mathcal{K}_{a_{3}}\left(2 b_{3} \sqrt{y}\right)$ \\
\hline $\begin{array}{l}\mu_{k, k 1}\left(y_{l_{1}} ; \theta, X, Z\right)=\left(\alpha_{S D} X \tau_{k}+\right. \\
\left.\left(1-\tau_{k 1}\right) \alpha_{R D} Z\right)\left(\theta-1+\theta y_{l_{1}}\right)\end{array}$ & $\xi_{3}\left(y ; \kappa_{1}, \kappa_{2}, \kappa_{3}, a_{1}, b_{1}\right)=2 X^{\kappa_{1}} Z^{\kappa_{2}} y^{\kappa_{3}} \mathcal{K}_{a_{1}}\left(2 b_{1} \sqrt{y}\right)$ \\
\hline $\begin{array}{l}\psi_{1, k 1}\left(\eta, y_{l_{1}}\right)=\alpha_{S D} \tau_{k 1}(\eta-1+ \\
\left.\eta y_{l_{1}}\right)+\left(1 / \Omega_{S P}\right)\end{array}$ & $\zeta_{2}\left(\eta, y_{l_{1}}, x_{l_{2}}\right)=2 \alpha_{S R} \sqrt{\eta-1+\eta y_{l_{1}}} \chi_{1, l_{1}, l_{2}} \mathcal{K}_{1}\left(2 \alpha_{S R} \sqrt{\eta-1+\eta y_{l_{1}}} \chi_{1, l_{1}, l_{2}}\right)$ \\
\hline $\begin{array}{l}\psi_{2, k 1}\left(\eta, y_{l_{1}}\right)=\alpha_{R D}\left(1-\tau_{k 1}\right)(\eta- \\
\left.1+\eta y_{l_{1}}\right)+\left(1 / \Omega_{R P}\right)\end{array}$ & $\psi_{1, k}\left(\eta, y_{l_{1}}\right)=\alpha_{S D} \tau_{k}\left(\eta-1+\eta y_{l_{1}}\right)+\left(1 / \Omega_{S P}\right)$ \\
\hline$\phi_{k 1}(\eta)=\varpi_{k 1}(\eta-1)+\left(1 / \Omega_{S P}\right)$ & $\bar{\xi}_{1}\left(y ; \kappa_{1}, \kappa_{2}, \kappa_{3}, a_{1}, a_{2}, b_{1}, b_{2}\right)=4 \chi_{1, l_{1}, l_{2}}^{2 \kappa_{1}} \chi_{2, l_{1}, l_{3}}^{2 \kappa_{2}} y^{\kappa_{3}} \mathcal{K}_{a_{1}}\left(2 b_{1} \sqrt{y}\right) \mathcal{K}_{a_{2}}\left(2 b_{2} \sqrt{y}\right)$ \\
\hline$\chi_{1, l_{1}, l_{2}}=\sqrt{\frac{x_{l_{2}}}{\psi_{1, k 1}\left(\eta, y_{l_{1}}\right)}}$ & $\bar{\xi}_{2}\left(y ; \kappa_{1}, \kappa_{2}, \kappa_{3}, a_{1}, a_{2}, a_{3}, b_{1}, b_{2}, b_{3}\right)=8 \chi_{1, l_{1}, l_{2}}^{2 \kappa_{1}} \chi_{2, l_{1}, l_{3}}^{2 \kappa_{2}} y^{\kappa_{3}} \mathcal{K}_{a_{1}}\left(2 b_{1} \sqrt{y}\right) \mathcal{K}_{a_{2}}\left(2 b_{2} \sqrt{y}\right) \mathcal{K}_{a_{3}}\left(2 b_{3} \sqrt{y}\right)$ \\
\hline$\chi_{2, l_{1}, l_{3}}=\sqrt{\frac{z_{l_{3}}}{\psi_{2, k 1}\left(\eta, y_{l_{1}}\right)}}$ & $\bar{\xi}_{3}\left(y ; \kappa_{1}, \kappa_{2}, \kappa_{3}, a_{1}, b_{1}\right)=2 \chi_{1, l_{1}, l_{2}}^{2 \kappa_{1}} \chi_{2, l_{1}, l_{3}}^{2 \kappa_{2}} y^{\kappa_{3}} \mathcal{K}_{a_{1}}\left(2 b_{1} \sqrt{y}\right)$ \\
\hline
\end{tabular}

As already observed that $\digamma_{D}$ and $\digamma_{E}$ are dependent due to the involved common channel gains $X$ and $Z$, hence, the first step of deriving the SOP is to derive the $\mathrm{CDF}$ of $\Theta$ conditioned on $X$ and $Z$, as shown in Theorem 1 .

Theorem 1: By the use of Proposition 2, the CDF of $\Theta$ conditioned on $X$ and $Z$, i.e., $F_{\Theta}^{\mathrm{LB}}(\theta \mid X, Z)$, can be given as

$$
\begin{aligned}
F_{\Theta}^{\mathrm{LB}}(\theta \mid X, Z) & =\sum_{k=1}^{M}\left[\left(\mathcal{S}_{1}(\theta \mid X, Z)-\mathcal{S}_{2}(\theta \mid X, Z)\right)\right. \\
& \left.-\left(\mathcal{S}_{3}(\theta \mid X, Z)-\mathcal{S}_{4}(\theta \mid X, Z)\right)\right],
\end{aligned}
$$

where the expressions $\mathcal{S}_{1}(\theta \mid X, Z)$ and $\mathcal{S}_{3}(\theta \mid X, Z)$ are given in (12) and (13), respectively, with $g_{l_{1}}=\frac{y_{l_{1}}}{\left(\left(U_{1}+1\right) L_{U_{1}+1}\left(y_{l_{1}}\right)\right)^{2}}$ and $y_{l_{1}},\left(l_{1}=1, \cdots, U_{1}\right)$ are the weights and zeros of $U_{1}-$ order Gauss-Laguerre polynomial (i.e., $L_{U_{1}}(y)$ ) [52, eq. (25.5.45)].

Moreover, $\mathcal{S}_{2}(\theta \mid X, Z)$ can be represented by replacing $\mathcal{C}_{1}(\theta, X)$ by $\mathcal{C}_{2}(\theta, X)$ and $\varpi_{k 1}$ by $\varpi_{k}$ in (12), and $\mathcal{S}_{4}(\theta \mid X, Z)$ can be expressed by replacing $\mu_{k 1}\left(y_{l_{1}} ; \theta, X, Z\right)$ with $\mu_{k, k 1}\left(y_{l_{1}} ; \theta, X, Z\right)$ in (13). The auxiliary functions used in $\mathcal{S}_{i}(\theta \mid X, Z)$, for $i \in\{1,2,3,4\}$ are given in Table III.

Proof: See Appendix $\mathrm{C}$ for the detailed analysis.

Remark 6: The conditional CDF obtained in Theorem 1 is dependent on $\mathcal{S}_{i}(\theta \mid X, Z)$, for $i \in\{1,2,3,4\}$, thus it can be noted that i) $\mathcal{S}_{1}(\theta \mid X, Z)$ in (12) is directly proportional to $\mathcal{C}_{1}(\theta, X)=\mathrm{e}^{-\frac{\tau_{k 1}(\theta-1) X}{\rho \Omega_{S D}}}$, and the other involved terms after simplification are observed independent of $\rho$, and $\mathcal{S}_{3}(\theta \mid X, Z)$ in (13) is directly proportional to $\frac{1}{\sqrt{\rho} \Omega_{S R}} \mathrm{e}^{-\left(\frac{X \tau_{k 1}}{\rho \Omega_{S D}}+\frac{\left(1-\tau_{k 1}\right) Z}{\rho \Omega_{R D}}\right)\left(\theta-1+\theta y_{l_{1}}\right)} \mathcal{K}_{1}\left(\frac{2 \sqrt{\left(\theta-1+\theta y_{l_{1}}\right) X}}{\sqrt{\rho} \Omega_{S R}}\right)$, and the other involved terms do not have significant impact of $\rho$, ii) as $\rho$ increases, $\mathrm{e}^{-\frac{\tau_{k 1}(\theta-1) X}{\rho \Omega_{S D}}}$ increases and hence $\mathcal{S}_{1}(\theta \mid X, Z)$, whereas $\mathcal{S}_{3}(\theta \mid X, Z)$ increases because of the dominance of increasing behavior of $\mathrm{e}^{-\left(\frac{X \tau_{k 1}}{\rho \Omega_{S D}}+\frac{\left(1-\tau_{k 1}\right) Z}{\rho \Omega_{R D}}\right)\left(\theta-1+\theta y l_{1}\right)}$ and $\mathcal{K}_{1}\left(\frac{2 \sqrt{\left(\theta-1+\theta y_{l_{1}}\right) X}}{\sqrt{\rho} \Omega_{S R}}\right)$ with $\rho$, iii) $\mathcal{S}_{3}(\theta \mid X, Z)$ increases more rapidly than $\mathcal{S}_{1}(\theta \mid X, Z)$ for all values of $\rho$, while ensuring $\mathcal{S}_{1}(\theta \mid X, Z)>\mathcal{S}_{3}(\theta \mid X, Z)$ (likewise, $\mathcal{S}_{2}(\theta \mid X, Z)>\mathcal{S}_{4}(\theta \mid X, Z)$ ). Also, $\mathcal{S}_{1}(\theta \mid X, Z)>$ $\mathcal{S}_{2}(\theta \mid X, Z)$ and $\mathcal{S}_{3}(\theta \mid X, Z)>\mathcal{S}_{4}(\theta \mid X, Z)$ hold true, iv) from (11), we infer that $\mathcal{S}_{1}(\theta \mid X, Z)-\mathcal{S}_{2}(\theta \mid X, Z)>\mathcal{S}_{3}(\theta \mid X, Z)-$ $\mathcal{S}_{4}(\theta \mid X, Z)$, and hence the conditional CDF decays as $\rho$ increases, v) the behavior of $F_{\Theta}^{\mathrm{LB}}(\theta \mid X, Z)$ for other channel parameters $\left(\Omega_{S D}, \Omega_{S R}, \Omega_{R D}, \Omega_{S E}\right.$, and $\left.\Omega_{R E}\right)$ can also be readily obtained, and vi) in Theorem 1 , convergence of $U_{1}$-order Gauss-Laguerre series and infinite series is given in Remark 10 and Remark 11 of Theorem 2, respectively.

Remark 7: It can also be noted that i) the derived conditional CDF, $F_{\Theta}^{\mathrm{LB}}(\theta \mid X, Z)$, in Theorem 1 can achieve its minimum for $\mathcal{S}_{1}(\theta \mid X, Z)=\mathcal{S}_{2}(\theta \mid X, Z)$ and $\mathcal{S}_{3}(\theta \mid X, Z)=\mathcal{S}_{4}(\theta \mid X, Z)$, and can be verified numerically, and ii) the derived $F_{\Theta}^{\mathrm{LB}}(\theta \mid X, Z)$ will be used to effectively obtain the required unconditional SOP of the considered system, as presented in Theorem 2 .

Theorem 2: By averaging Theorem 1 over $X$ and $Z$, the lower bound of the SOP can be expressed as

$\mathcal{P}_{\text {sec }}^{\text {out LB }}(\eta)=\sum_{k=1}^{M}\left[\left(\mathcal{S}_{1}(\eta)-\mathcal{S}_{2}(\eta)\right)-\left(\mathcal{S}_{3}(\eta)-\mathcal{S}_{4}(\eta)\right)\right]$,

where $\mathcal{S}_{1}(\eta)$ is given in (15), with $g_{l_{2}}=\frac{x_{l_{2}}}{\left(\left(U_{2}+1\right) L_{U_{2}+1}\left(x_{l_{2}}\right)\right)^{2}}$ and $x_{l_{2}},\left(l_{2}=1, \cdots, U_{2}\right)$ are weights and zeros of $U_{2}$-order Gauss-Laguerre polynomial (i.e., $L_{U_{2}}(x)$ ), and $g_{l_{3}}=\frac{z_{l_{3}}}{\left(\left(U_{3}+1\right) L_{U_{3}+1}\left(z_{l_{3}}\right)\right)^{2}}$ and $z_{l_{3}},\left(l_{3}=1, \cdots, U_{3}\right)$ are weights and zeros of $U_{3}$-order Gauss-Laguerre polynomial (i.e., $\left.L_{U_{3}}(z)\right)$ [52, eq. (25.5.45)]. Moreover, $\mathcal{S}_{2}(\eta)$ in (14) can be evaluated and represented by replacing $\varpi_{k 1}$ by $\varpi_{k}$ in (15).

Further, $\mathcal{S}_{3}(\eta)$ is presented in (16). And, $\mathcal{S}_{4}(\eta)$ in (14) can be expressed by replacing $\psi_{1, k 1}\left(\eta, y_{l_{1}}\right)$ by $\psi_{1, k}\left(\eta, y_{l_{1}}\right)$ in (16). The auxiliary functions used in (14) are given in Table III.

Finally, by invoking the results of $\mathcal{S}_{1}(\eta), \mathcal{S}_{2}(\eta), \mathcal{S}_{3}(\eta)$, and $\mathcal{S}_{4}(\eta)$ into (14), we can obtain the tight lower bound SOP 


$$
\begin{aligned}
& \mathcal{S}_{1}(\theta \mid X, Z)=\mathcal{C}_{1}(\theta, X)\left\{\frac{Z}{X} \frac{\alpha_{R E}^{2}}{\alpha_{S R}^{2}} \mathcal{N}_{1}\left(\frac{\varpi_{k 1} \theta}{\alpha_{S R}^{2}}, \frac{Z}{X} \frac{\alpha_{R E}^{2}}{\alpha_{S R}^{2}}\right)+\frac{X}{Z} \frac{\alpha_{S R}^{2}}{\alpha_{R E}^{2}} \mathcal{N}_{1}\left(\frac{\varpi_{k 1} \theta X}{Z \alpha_{R E}^{2}}, \frac{X}{Z} \frac{\alpha_{S R}^{2}}{\alpha_{R E}^{2}}\right)-\sum_{u=0}^{\infty} \frac{(-1)^{u}\left(\varpi_{k 1} \theta X\right)^{u}}{u !}\right. \\
& \times\left(\frac{M}{X \alpha_{S E}^{2}}\right)^{u+2}\left[\frac{\alpha_{S R} \alpha_{R E}^{2} \alpha_{S E} X Z}{\sqrt{M}} \mathcal{N}_{2}\left(-\frac{3}{2}-u,-\frac{1}{2}-u, 0,0, \frac{1}{2},-\frac{1}{2}, \frac{M Z}{X}, M\right)+\frac{\alpha_{S R}^{2} \alpha_{R E} \alpha_{S E} X^{\frac{3}{2}} \sqrt{Z}}{\sqrt{M}}\right. \\
& \left.\times \mathcal{N}_{2}\left(-\frac{3}{2}-u,-\frac{1}{2}-u, \frac{1}{2},-\frac{1}{2}, 0,0, \frac{M Z}{X}, M\right)+\frac{\alpha_{S R} \alpha_{R E} \alpha_{S E}^{2} X^{\frac{3}{2}} \sqrt{Z}}{M} \mathcal{N}_{2}\left(-1-u,-1-u, \frac{1}{2},-\frac{1}{2}, \frac{1}{2},-\frac{1}{2}, \frac{M Z}{X}, M\right)\right] \\
& +\frac{\alpha_{S E}}{\sqrt{M \varpi_{k 1} \theta}} \mathrm{e}^{\frac{1}{2} \frac{\alpha_{S E}^{2}}{M \varpi_{k 1} \theta}} \mathcal{W}_{-\frac{1}{2}, 0}\left(\frac{\alpha_{S E}^{2}}{M \varpi_{k 1} \theta}\right)+\sum_{j=2}^{M}\left(\frac{\alpha_{S E}}{\sqrt{\varpi_{k 1} \theta}}\left[-\sqrt{\tau_{j 1}} \mathrm{e}^{\frac{1}{2} \frac{\alpha_{S E}^{2} \tau_{j 1}}{\varpi_{k 1} \theta}} \mathcal{W}_{-\frac{1}{2}, 0}\left(\frac{\alpha_{S E}^{2} \tau_{j 1}}{\varpi_{k 1} \theta}\right)+\sqrt{\tau_{j}} \mathrm{e}^{\frac{1}{2} \frac{\alpha_{S E}^{2} \tau_{j}}{\varpi_{k 1} \theta}} \mathcal{W}_{-\frac{1}{2}, 0}\left(\frac{\alpha_{S E}^{2} \tau_{j}}{\varpi_{k 1} \theta}\right)\right]\right. \\
& +\sum_{u=0}^{\infty} \frac{(-1)^{u}\left(\varpi_{k 1} \theta X\right)^{u}}{u !}\left[\frac{\alpha_{S R} \alpha_{R E}\left(1-\tau_{j 1}\right) \sqrt{Z}}{X^{u+\frac{1}{2}}\left(\alpha_{S E}^{2} \tau_{j 1}\right)^{u+1}} \mathcal{N}_{2}\left(-1-u,-1-u, \frac{1}{2},-\frac{1}{2}, \frac{1}{2},-\frac{1}{2}, \frac{\left(1-\tau_{j 1}\right) Z}{\tau_{j 1} X}, \frac{1-\tau_{j 1}}{\tau_{j 1}}\right)\right. \\
& -\frac{\alpha_{S R} \alpha_{R E}\left(1-\tau_{j 1}\right) \sqrt{Z}}{X^{u+\frac{1}{2}}\left(\alpha_{S E}^{2} \tau_{j}\right)^{u+1}} \mathcal{N}_{2}\left(-1-u,-1-u, \frac{1}{2},-\frac{1}{2}, \frac{1}{2},-\frac{1}{2}, \frac{\left(1-\tau_{j 1}\right) Z}{\tau_{j} X}, \frac{1-\tau_{j 1}}{\tau_{j}}\right) \\
& +\frac{\alpha_{S R} \alpha_{R E}^{2} Z\left(1-\tau_{j 1}\right)^{\frac{3}{2}}}{X^{u+1}\left(\alpha_{S E}^{2} \tau_{j 1}\right)^{u+\frac{3}{2}}} \mathcal{N}_{2}\left(-\frac{3}{2}-u,-\frac{1}{2}-u, 0,0, \frac{1}{2},-\frac{1}{2}, \frac{\left(1-\tau_{j 1}\right) Z}{\tau_{j 1} X}, \frac{1-\tau_{j 1}}{\tau_{j 1}}\right) \\
& -\frac{\alpha_{S R} \alpha_{R E}^{2} Z\left(1-\tau_{j 1}\right)^{\frac{3}{2}}}{X^{u+1}\left(\alpha_{S E}^{2} \tau_{j}\right)^{u+\frac{3}{2}}} \mathcal{N}_{2}\left(-\frac{3}{2}-u,-\frac{1}{2}-u, 0,0, \frac{1}{2},-\frac{1}{2}, \frac{\left(1-\tau_{j 1}\right) Z}{\tau_{j} X}, \frac{1-\tau_{j 1}}{\tau_{j}}\right) \\
& +\frac{\alpha_{S R}^{2} \alpha_{R E} \sqrt{Z}\left(1-\tau_{j 1}\right)^{\frac{3}{2}}}{X^{u+\frac{1}{2}}\left(\alpha_{S E}^{2} \tau_{j 1}\right)^{u+\frac{3}{2}}} \mathcal{N}_{2}\left(-\frac{3}{2}-u,-\frac{1}{2}-u, \frac{1}{2},-\frac{1}{2}, 0,0, \frac{\left(1-\tau_{j 1}\right) Z}{\tau_{j 1} X}, \frac{1-\tau_{j 1}}{\tau_{j 1}}\right) \\
& \left.\left.\left.-\frac{\alpha_{S R}^{2} \alpha_{R E} \sqrt{Z}\left(1-\tau_{j 1}\right)^{\frac{3}{2}}}{X^{u+\frac{1}{2}}\left(\alpha_{S E}^{2} \tau_{j}\right)^{u+\frac{3}{2}}} \mathcal{N}_{2}\left(-\frac{3}{2}-u,-\frac{1}{2}-u, \frac{1}{2},-\frac{1}{2}, 0,0, \frac{\left(1-\tau_{j 1}\right) Z}{\tau_{j 1} X}, \frac{1-\tau_{j 1}}{\tau_{j 1}}\right)\right]\right)\right\},
\end{aligned}
$$

$\mathcal{S}_{3}(\theta \mid X, Z)=\sum_{l_{1}=1}^{U_{1}} g_{l_{1}} \mathrm{e}^{-\mu_{k 1}\left(y_{l_{1}} ; \theta, X, Z\right)} \mathrm{e}^{y_{l_{1}}} \zeta_{1}\left(y_{l_{1}} ; \theta, X\right)\left\{\alpha_{S R} \alpha_{R E}^{2} \xi_{1}\left(y_{l_{1}} ; \frac{1}{2}, 1, \frac{1}{2}, 0,1, \alpha_{R E} \sqrt{Z}, \alpha_{S R} \sqrt{X}\right)+\alpha_{S R}^{2} \alpha_{R E}\right.$

$\times \xi_{1}\left(y_{l_{1}} ; 1, \frac{1}{2}, \frac{1}{2}, 1,0, \alpha_{R E} \sqrt{Z}, \alpha_{S R} \sqrt{X}\right)-\frac{\alpha_{S R} \alpha_{R E}^{2} \alpha_{S E}}{\sqrt{M}} \xi_{2}\left(y_{l_{1}} ; 1,1,1,1,0,1, \frac{\alpha_{S E} \sqrt{X}}{\sqrt{M}}, \alpha_{R E} \sqrt{Z}, \alpha_{S R} \sqrt{X}\right)-\frac{\alpha_{S R}^{2} \alpha_{R E} \alpha_{S E}}{\sqrt{M}}$ $\times \xi_{2}\left(y_{l_{1}} ; \frac{3}{2}, \frac{1}{2}, 1,1,1,0, \frac{\alpha_{S E} \sqrt{X}}{\sqrt{M}}, \alpha_{R E} \sqrt{Z}, \alpha_{S R} \sqrt{X}\right)-\frac{\alpha_{S R} \alpha_{R E} \alpha_{S E}^{2}}{M} \xi_{2}\left(y_{l_{1}} ; \frac{3}{2}, \frac{1}{2}, 1,0,1,1, \frac{\alpha_{S E} \sqrt{X}}{\sqrt{M}}, \alpha_{R E} \sqrt{Z}, \alpha_{S R} \sqrt{X}\right)$ $+\frac{\alpha_{S R} \alpha_{S E}^{2}}{M \alpha_{S R}} \xi_{3}\left(y_{l_{1}} ; 1,0,0,0, \frac{\alpha_{S E} \sqrt{X}}{\sqrt{M}}\right)+\sum_{j=2}^{M}\left[-\alpha_{S E}^{2} \tau_{j 1} \xi_{3}\left(y_{l_{1}} ; 1,0,0,0, \alpha_{S E} \tau_{j 1}^{\frac{1}{2}} \sqrt{X}\right)+\alpha_{S E}^{2} \tau_{j} \xi_{3}\left(y_{l_{1}} ; 1,0,0,0, \alpha_{S E} \tau_{j}^{\frac{1}{2}} \sqrt{X}\right)\right.$ $+\alpha_{S R} \alpha_{R E} \alpha_{S E}^{2} \tau_{j 1}\left(1-\tau_{j 1}\right) \xi_{2}\left(y_{l_{1}} ; \frac{3}{2}, \frac{1}{2}, 1,0,1,1, \alpha_{S E} \tau_{j 1}^{\frac{1}{2}} \sqrt{X}, \alpha_{R E} \sqrt{Z}\left(1-\tau_{j 1}\right)^{\frac{1}{2}}, \alpha_{S R} \sqrt{X}\left(1-\tau_{j 1}\right)^{\frac{1}{2}}\right)-\alpha_{S R} \alpha_{R E} \alpha_{S E}^{2}\left(1-\tau_{j 1}\right)$ $\times \tau_{j} \xi_{2}\left(y_{l_{1}} ; \frac{3}{2}, \frac{1}{2}, 1,0,1,1, \alpha_{S E} \tau_{j}^{\frac{1}{2}} \sqrt{X}, \alpha_{R E}\left(1-\tau_{j 1}\right)^{\frac{1}{2}} \sqrt{Z}, \alpha_{S R}\left(1-\tau_{j 1}\right)^{\frac{1}{2}} \sqrt{X}\right)+\alpha_{S R} \alpha_{R E}^{2} \alpha_{S E} \tau_{j 1}^{\frac{1}{2}}\left(1-\tau_{j 1}\right)^{\frac{3}{2}} \xi_{2}\left(y_{l_{1}} ; 1,1,1\right.$ $\left., 1,0,1, \alpha_{S E} \tau_{j 1}^{\frac{1}{2}} \sqrt{X}, \alpha_{R E}\left(1-\tau_{j 1}\right)^{\frac{1}{2}} \sqrt{Z}, \alpha_{S R}\left(1-\tau_{j 1}\right)^{\frac{1}{2}} \sqrt{X}\right)-\alpha_{S R} \alpha_{R E}^{2} \alpha_{S E} \tau_{j}^{\frac{1}{2}}\left(1-\tau_{j 1}\right)^{\frac{3}{2}} \xi_{2}\left(y_{l_{1}} ; 1,1,1,1,0,1, \alpha_{S E} \tau_{j}^{\frac{1}{2}} \sqrt{X}\right.$ $\left., \alpha_{R E}\left(1-\tau_{j 1}\right)^{\frac{1}{2}} \sqrt{Z}, \alpha_{S R}\left(1-\tau_{j 1}\right)^{\frac{1}{2}} \sqrt{X}\right)+\alpha_{S R}^{2} \alpha_{R E} \alpha_{S E} \tau_{j 1}^{\frac{1}{2}}\left(1-\tau_{j 1}\right)^{\frac{3}{2}} \xi_{2}\left(y_{l_{1}} ; \frac{3}{2}, \frac{1}{2}, 1,1,1,0, \alpha_{S E} \tau_{j 1}^{\frac{1}{2}} \sqrt{X}, \alpha_{R E}\left(1-\tau_{j 1}\right)^{\frac{1}{2}} \sqrt{Z}\right.$ ,$\left.\left.\left.\alpha_{S R}\left(1-\tau_{j 1}\right)^{\frac{1}{2}} \sqrt{X}\right)-\alpha_{S R}^{2} \alpha_{R E} \alpha_{S E} \tau_{j}^{\frac{1}{2}}\left(1-\tau_{j 1}\right)^{\frac{3}{2}} \xi_{2}\left(y_{l_{1}} ; \frac{3}{2}, \frac{1}{2}, 1,1,1,0, \alpha_{S E} \tau_{j}^{\frac{1}{2}} \sqrt{X}, \alpha_{R E}\left(1-\tau_{j 1}\right)^{\frac{1}{2}} \sqrt{Z}, \alpha_{S R}\left(1-\tau_{j 1}\right)^{\frac{1}{2}} \sqrt{X}\right)\right]\right\}$,

expression over mixed Rayleigh and double-Rayleigh fading.

Proof: See Appendix D for the detailed proof.

Remark 8: The SOP analysis presented in Theorem 2 over mixed Rayleigh and double-Rayleigh fading channels has not yet been analyzed in the existing literature. It is worth noting that i) (15) and (16) look complex, however, by observing closely we can infer that they mainly involve powers, exponentials, modified Bessel functions of second kind, Whittaker$\mathcal{W}$ functions, and extended generalized bi-variate Meijer- $G$ functions ${ }^{4}$ consisting of system/channel parameters, such as,

${ }^{4}$ It can readily be implemented efficiently using Mathematica computation software package via [53]. 
$\mathcal{S}_{1}(\eta)=\frac{1}{\Omega_{S P} \phi_{k 1}(\eta)} \frac{\alpha_{S E}}{\sqrt{\varpi_{k 1} \eta}}\left[\frac{1}{\sqrt{M}} \mathrm{e}^{\frac{1}{2} \frac{\alpha_{S E}^{2}}{M \varpi_{k 1} \eta}} \mathcal{W}_{-\frac{1}{2}, 0}\left(\frac{\alpha_{S E}^{2}}{M \varpi_{k 1} \eta}\right)+\sum_{j=2}^{M}\left(-\sqrt{\tau_{j 1}} \mathrm{e}^{\frac{1}{2} \frac{\alpha_{S E}^{2} \tau_{j 1}}{\varpi_{k 1} \eta}} \mathcal{W}_{-\frac{1}{2}, 0}\left(\frac{\alpha_{S E}^{2} \tau_{j 1}}{\varpi_{k 1} \eta}\right)+\sqrt{\tau_{j}} \mathrm{e}^{\frac{1}{2} \frac{\alpha_{S E}^{2} \tau_{j}}{\varpi_{k 1} \eta}}\right.\right.$ $\left.\left.\times \mathcal{W}_{-\frac{1}{2}, 0}\left(\frac{\alpha_{S E}^{2} \tau_{j}}{\varpi_{k 1} \eta}\right)\right)\right]+\frac{1}{\Omega_{S P} \phi_{k 1}(\eta)} \sum_{l_{2}=1}^{U_{2}} \sum_{l_{3}=1}^{U_{3}} g_{l_{2}} g_{l_{3}}\left\{\frac{\phi_{k 1}(\eta) \Omega_{R P} z_{l_{3}}}{x_{l_{2}}} \frac{\alpha_{R E}^{2}}{\alpha_{S R}^{2}} \mathcal{N}_{1}\left(\frac{\varpi_{k 1} \eta}{\alpha_{S R}^{2}}, \frac{\phi_{k 1}(\eta) \Omega_{R P} z_{l_{3}}}{x_{l_{2}}} \frac{\alpha_{R E}^{2}}{\alpha_{S R}^{2}}\right)+\frac{x_{l_{2}}}{\phi_{k 1}(\eta) \Omega_{R P} z_{l_{3}}}\right.$ $\times \frac{\alpha_{S R}^{2}}{\alpha_{R E}^{2}} \mathcal{N}_{1}\left(\frac{\varpi_{k 1} \eta x_{l_{2}}}{\phi_{k 1}(\eta) \Omega_{R P} z_{l_{3}} \alpha_{R E}^{2}}, \frac{x_{l_{2}}}{\phi_{k 1}(\eta) \Omega_{R P} z_{l_{3}}} \frac{\alpha_{S R}^{2}}{\alpha_{R E}^{2}}\right)-\sum_{u=0}^{\infty} \frac{(-1)^{u}\left(\varpi_{k 1} \eta x_{l_{2}}\right)^{u}}{u !\left(\phi_{k 1}(\eta)\right)^{u}}\left(\frac{\phi_{k 1}(\eta) M}{x_{l_{2}} \alpha_{S E}^{2}}\right)^{u+2}\left[\frac{\alpha_{S R} \alpha_{R E}^{2} \alpha_{S E} \Omega_{R P} x_{l_{2}} z_{l_{3}}}{\sqrt{M} \phi_{k 1}(\eta)}\right.$ $\times \mathcal{N}_{2}\left(-\frac{3}{2}-u,-\frac{1}{2}-u, 0,0, \frac{1}{2},-\frac{1}{2}, \frac{M \phi_{k 1}(\eta) \Omega_{R P} z_{l_{3}}}{x_{l_{2}}}, M\right)+\frac{\alpha_{S R}^{2} \alpha_{R E} \alpha_{S E} x_{l_{2}}^{\frac{3}{2}} \sqrt{\Omega_{R P} z_{l_{3}}}}{\sqrt{M}\left(\phi_{k 1}(\eta)\right)^{\frac{3}{2}}} \mathcal{N}_{2}\left(-\frac{3}{2}-u,-\frac{1}{2}-u, \frac{1}{2},-\frac{1}{2}\right.$ $\left.\left., 0,0, \frac{M z_{l_{3}}}{x_{l_{2}}}, M\right)+\frac{\alpha_{S R} \alpha_{R E} \alpha_{S E}^{2} x_{l_{2}}^{\frac{3}{2}} \sqrt{\Omega_{R P} z_{l_{3}}}}{M\left(\phi_{k 1}(\eta)\right)^{\frac{3}{2}}} \mathcal{N}_{2}\left(-1-u,-1-u, \frac{1}{2},-\frac{1}{2}, \frac{1}{2},-\frac{1}{2}, \frac{M \phi_{k 1}(\eta) \Omega_{R P} z_{l_{3}}}{x_{l_{2}}}, M\right)\right]+\sum_{j=2}^{M} \sum_{u=0}^{\infty} \frac{(-1)^{u}}{u !}$ $\times\left(\varpi_{k 1} \eta x_{l_{2}}\right)^{u}\left[\frac{\alpha_{S R} \alpha_{R E}\left(1-\tau_{j 1}\right)\left(\phi_{k 1}(\eta)\right)^{\frac{1}{2}} \sqrt{\Omega_{R P} z_{l_{3}}}}{x_{l_{2}}^{u+\frac{1}{2}}\left(\alpha_{S E}^{2} \tau_{j 1}\right)^{u+1}} \mathcal{N}_{2}\left(-1-u,-1-u, \frac{1}{2},-\frac{1}{2}, \frac{1}{2},-\frac{1}{2}, \frac{\left(1-\tau_{j 1}\right) \phi_{k 1}(\eta) \Omega_{R P} z_{l_{3}}}{\tau_{j 1} x_{l_{2}}}, \frac{1-\tau_{j 1}}{\tau_{j 1}}\right)\right.$ $-\frac{\alpha_{S R} \alpha_{R E}\left(1-\tau_{j 1}\right)\left(\phi_{k 1}(\eta)\right)^{\frac{1}{2}} \sqrt{\Omega_{R P} z_{l_{3}}}}{x_{l_{2}}^{u+\frac{1}{2}}\left(\alpha_{S E}^{2} \tau_{j}\right)^{u+1}} \mathcal{N}_{2}\left(-1-u,-1-u, \frac{1}{2},-\frac{1}{2}, \frac{1}{2},-\frac{1}{2}, \frac{\left(1-\tau_{j 1}\right) \phi_{k 1}(\eta) \Omega_{R P} z_{l_{3}}}{\tau_{j} x_{l_{2}}}, \frac{1-\tau_{j 1}}{\tau_{j}}\right)$ $+\frac{\alpha_{S R} \alpha_{R E}^{2}\left(1-\tau_{j 1}\right)^{\frac{3}{2}} \phi_{k 1}(\eta) \Omega_{R P} z_{l_{3}}}{x_{l_{2}}^{u+1}\left(\alpha_{S E}^{2} \tau_{j 1}\right)^{u+\frac{3}{2}}} \mathcal{N}_{2}\left(-\frac{3}{2}-u,-\frac{1}{2}-u, 0,0, \frac{1}{2},-\frac{1}{2}, \frac{\left(1-\tau_{j 1}\right) \phi_{k 1}(\eta) \Omega_{R P} z_{l_{3}}}{\tau_{j 1} x_{l_{2}}}, \frac{1-\tau_{j 1}}{\tau_{j 1}}\right)$ $-\frac{\alpha_{S R} \alpha_{R E}^{2}\left(1-\tau_{j 1}\right)^{\frac{3}{2}} \phi_{k 1}(\eta) \Omega_{R P} z_{l_{3}}}{x_{l_{2}}^{u+1}\left(\alpha_{S E}^{2} \tau_{j}\right)^{u+\frac{3}{2}}} \mathcal{N}_{2}\left(-\frac{3}{2}-u,-\frac{1}{2}-u, 0,0, \frac{1}{2},-\frac{1}{2}, \frac{\left(1-\tau_{j 1}\right) \phi_{k 1}(\eta) \Omega_{R P} z_{l_{3}}}{\tau_{j} x_{l_{2}}}, \frac{1-\tau_{j 1}}{\tau_{j}}\right)$ $+\frac{\alpha_{S R}^{2} \alpha_{R E}\left(1-\tau_{j 1}\right)^{\frac{3}{2}}\left(\phi_{k 1}(\eta)\right)^{\frac{1}{2}} \sqrt{\Omega_{R P} z_{l_{3}}}}{x_{l_{2}}^{u+\frac{1}{2}}\left(\alpha_{S E}^{2} \tau_{j 1}\right)^{u+\frac{3}{2}}} \mathcal{N}_{2}\left(-\frac{3}{2}-u,-\frac{1}{2}-u, \frac{1}{2},-\frac{1}{2}, 0,0, \frac{\left(1-\tau_{j 1}\right) \phi_{k 1}(\eta) \Omega_{R P} z_{l_{3}}}{\tau_{j 1} x_{l_{2}}}, \frac{1-\tau_{j 1}}{\tau_{j 1}}\right)$ $\left.\left.-\frac{\alpha_{S R}^{2} \alpha_{R E}\left(1-\tau_{j 1}\right)^{\frac{3}{2}}\left(\phi_{k 1}(\eta)\right)^{\frac{1}{2}} \sqrt{\Omega_{R P} z_{l_{3}}}}{x_{l_{2}}^{u+\frac{1}{2}}\left(\alpha_{S E}^{2} \tau_{j}\right)^{u+\frac{3}{2}}} \mathcal{N}_{2}\left(-\frac{3}{2}-u,-\frac{1}{2}-u, \frac{1}{2},-\frac{1}{2}, 0,0, \frac{\left(1-\tau_{j 1}\right) \phi_{k 1}(\eta) \Omega_{R P} z_{l_{3}}}{\tau_{j 1} x_{l_{2}}}, \frac{1-\tau_{j 1}}{\tau_{j 1}}\right)\right]\right\}$,

$\mathcal{S}_{3}(\eta)=\sum_{l_{1}=1}^{U_{1}} \sum_{l_{2}=1}^{U_{2}} \sum_{l_{3}=1}^{U_{3}} \frac{g_{l_{1}} g_{l_{2}} g_{l_{3}} \zeta_{2}\left(\eta, y_{l_{1}}, x_{l_{2}}\right) \mathrm{e}^{y_{l_{1}}} \alpha_{S R}}{\Omega_{S P} \Omega_{R P} \psi_{1, k 1}\left(\eta, y_{l_{1}}\right) \psi_{2, k 1}\left(\eta, y_{l_{1}}\right)}\left\{\alpha_{R E}^{2} \bar{\xi}_{1}\left(y_{l_{1}} ; \frac{1}{2}, 1, \frac{1}{2}, 0,1, \alpha_{R E} \chi_{2, l_{1}, l_{3}}, \alpha_{S R} \chi_{1, l_{1}, l_{2}}\right)+\alpha_{S R} \alpha_{R E}\right.$ $\times \bar{\xi}_{1}\left(y_{l_{1}} ; 1, \frac{1}{2}, \frac{1}{2}, 1,0, \alpha_{R E} \chi_{2, l_{1}, l_{3}}, \alpha_{S R} \chi_{1, l_{1}, l_{2}}\right)-\frac{\alpha_{S R} \alpha_{R E}^{2} \alpha_{S E}}{\sqrt{M}} \bar{\xi}_{2}\left(y_{l_{1}} ; 1,1,1,1,0,1, \frac{\alpha_{S E}}{\sqrt{M}} \chi_{1, l_{1}, l_{2}}, \alpha_{R E} \chi_{2, l_{1}, l_{3}}, \alpha_{S R} \chi_{1, l_{1}, l_{2}}\right)$ $-\frac{\alpha_{S R} \alpha_{R E} \alpha_{S E}}{\sqrt{M}} \bar{\xi}_{2}\left(y_{l_{1}} ; \frac{3}{2}, \frac{1}{2}, 1,1,1,0, \frac{\alpha_{S E}}{\sqrt{M}} \chi_{1, l_{1}, l_{2}}, \alpha_{R E} \chi_{2, l_{1}, l_{3}}, \alpha_{S R} \chi_{1, l_{1}, l_{2}}\right)-\frac{\alpha_{R E} \alpha_{S E}^{2}}{M} \bar{\xi}_{2}\left(y_{l_{1}} ; \frac{3}{2}, \frac{1}{2}, 1,0,1,1, \frac{\alpha_{S E}}{\sqrt{M}} \chi_{1, l_{1}, l_{2}}\right.$ $\left., \alpha_{R E} \chi_{2, l_{1}, l_{3}}, \alpha_{S R} \chi_{1, l_{1}, l_{2}}\right)+\frac{\alpha_{S E}^{2}}{M \alpha_{S R}} \bar{\xi}_{3}\left(y_{l_{1}} ; 1,0,0,0, \frac{\alpha_{S E}}{\sqrt{M}} \chi_{1, l_{1}, l_{2}}\right)+\sum_{j=2}^{M}\left[-\frac{\alpha_{S E}^{2} \tau_{j 1}}{\alpha_{S R}} \bar{\xi}_{3}\left(y_{l_{1}} ; 1,0,0,0, \alpha_{S E} \sqrt{\tau_{j 1}} \chi_{1, l_{1}, l_{2}}\right)\right.$ $+\frac{\alpha_{S E}^{2} \tau_{j}}{\alpha_{S R}} \bar{\xi}_{3}\left(y_{l_{1}} ; 1,0,0,0, \alpha_{S E} \sqrt{\tau_{j}} \chi_{1, l_{1}, l_{2}}\right)+\alpha_{S R} \alpha_{R E} \alpha_{S E}^{2} \tau_{j 1}\left(1-\tau_{j 1}\right) \bar{\xi}_{2}\left(y_{l_{1}} ; \frac{3}{2}, \frac{1}{2}, 1,0,1,1, \alpha_{S E} \sqrt{\tau_{j 1}} \chi_{1, l_{1}, l_{2}}\right.$ $\left., \alpha_{R E}\left(1-\tau_{j 1}\right)^{\frac{1}{2}} \chi_{2, l_{1}, l_{3}}, \alpha_{S R}\left(1-\tau_{j 1}\right)^{\frac{1}{2}} \chi_{1, l_{1}, l_{2}}\right)-\alpha_{R E} \alpha_{S E}^{2} \tau_{j}\left(1-\tau_{j 1}\right) \bar{\xi}_{2}\left(y_{l_{1}} ; \frac{3}{2}, \frac{1}{2}, 1,0,1,1, \alpha_{S E} \sqrt{\tau_{j}} \chi_{1, l_{1}, l_{2}}\right.$ $\left., \alpha_{R E}\left(1-\tau_{j 1}\right)^{\frac{1}{2}} \chi_{2, l_{1}, l_{3}}, \alpha_{S R}\left(1-\tau_{j 1}\right)^{\frac{1}{2}} \chi_{1, l_{1}, l_{2}}\right)+\alpha_{R E}^{2} \alpha_{S E} \tau_{j 1}^{\frac{1}{2}}\left(1-\tau_{j 1}\right)^{\frac{3}{2}} \bar{\xi}_{2}\left(y_{l_{1}} ; 1,1,1,1,0,1, \alpha_{S E} \sqrt{\tau_{j 1}} \chi_{1, l_{1}, l_{2}}\right.$ $\left., \alpha_{R E}\left(1-\tau_{j 1}\right)^{\frac{1}{2}} \chi_{2, l_{1}, l_{3}}, \alpha_{S R}\left(1-\tau_{j 1}\right)^{\frac{1}{2}} \chi_{1, l_{1}, l_{2}}\right)-\alpha_{R E}^{2} \alpha_{S E} \tau_{j}^{\frac{1}{2}}\left(1-\tau_{j 1}\right)^{\frac{3}{2}} \bar{\xi}_{2}\left(y_{l_{1}} ; 1,1,1,1,0,1, \alpha_{S E} \sqrt{\tau_{j}} \chi_{1, l_{1}, l_{2}}\right.$ $\left., \alpha_{R E}\left(1-\tau_{j 1}\right)^{\frac{1}{2}} \chi_{2, l_{1}, l_{3}}, \alpha_{S R}\left(1-\tau_{j 1}\right)^{\frac{1}{2}} \chi_{1, l_{1}, l_{2}}\right)+\alpha_{S R} \alpha_{R E} \alpha_{S E} \tau_{j 1}^{\frac{1}{2}}\left(1-\tau_{j 1}\right)^{\frac{3}{2}} \bar{\xi}_{2}\left(y_{l_{1}} ; \frac{3}{2}, \frac{1}{2}, 1,1,1,0, \alpha_{S E} \sqrt{\tau_{j 1}} \chi_{1, l_{1}, l_{2}}\right.$ $\left., \alpha_{R E}\left(1-\tau_{j 1}\right)^{\frac{1}{2}} \chi_{2, l_{1}, l_{3}}, \alpha_{S R}\left(1-\tau_{j 1}\right)^{\frac{1}{2}} \chi_{1, l_{1}, l_{2}}\right)-\alpha_{S R} \alpha_{R E} \alpha_{S E} \tau_{j}^{\frac{1}{2}}\left(1-\tau_{j 1}\right)^{\frac{3}{2}}$

$\left.\left.\times \bar{\xi}_{2}\left(y_{l_{1}} ; \frac{3}{2}, \frac{1}{2}, 1,1,1,0, \alpha_{S E} \sqrt{\tau_{j}} \chi_{1, l_{1}, l_{2}}, \alpha_{R E}\left(1-\tau_{j 1}\right)^{\frac{1}{2}} \chi_{2, l_{1}, l_{3}}, \alpha_{S R}\left(1-\tau_{j 1}\right)^{\frac{1}{2}} \chi_{1, l_{1}, l_{2}}\right)\right]\right\}$ 
$\mathcal{Q}, N_{0}, \Omega_{S D}, \Omega_{S R}, \Omega_{R D}, \Omega_{S P}, \Omega_{R P}, \Omega_{S E}$, and $\Omega_{R E}$, ii) for all the values of system/channel parameters, we have $\mathcal{S}_{1}(\eta)-$ $\mathcal{S}_{2}(\eta)>\mathcal{S}_{3}(\eta)-\mathcal{S}_{4}(\eta)$, where the increasing/decreasing trend of $\mathcal{S}_{i}(\eta)$, for $i \in\{1,2,3,4\}$ will decide the SOP behavior, as shown numerically in Section IV, and iii) the minimum SOP, i.e., $\mathcal{P}_{\text {sec }}^{\text {out, } \mathrm{LB}}(\eta)=0$ can be theoretically attained when $\mathcal{S}_{1}(\eta)=\mathcal{S}_{2}(\eta)$ and $\mathcal{S}_{3}(\eta)=\mathcal{S}_{4}(\eta)$, which can be obtained numerically for the involved parameters in Section IV.

Remark 9: From Theorem 2, we can infer the following SOP performance trends: i) the SOP improves as $\Omega_{S D}, \Omega_{S R}$, and $\Omega_{R D}$ increase, ii) the improvement in eavesdropper channel quality leads to a poorer SOP performance, iii) the SOP performance decreases when the strength of interference channels (i.e., $\Omega_{S P}$ and $\Omega_{R P}$ ) increase, and iv) the SOP saturates as $\rho$ increases, because $\rho$ at both $D$ and $E$ increase simultaneously. These trends are further verified numerically in Section IV.

Remark 10: The SOP expression in Theorem 2 consists of $U_{1}, U_{2}$, and $U_{3}$ order Gauss-Laguerre series expansions, which are convergent and make them of practical use. For instance, consider a term from (16), i.e., $\mathcal{Z}=\sum_{l_{1}=1}^{U_{1}} \sum_{l_{2}=1}^{U_{2}} \sum_{l_{3}=1}^{U_{3}} \frac{g_{l_{1}} g_{l_{2}} g_{l_{3}} \zeta_{2}\left(\eta, y_{l_{1}}, x_{l_{2}}\right) \mathrm{e}^{y_{l_{1}}} \alpha_{S R} \alpha_{R E}^{2}}{\Omega_{S P} \Omega_{R P} \psi_{1, k 1}\left(\eta, y_{l_{1}}\right) \psi_{2, k 1}\left(\eta, y_{l_{1}}\right)}$ $\times \quad \bar{\xi}_{1}\left(y_{l_{1}} ; \frac{1}{2}, 1, \frac{1}{2}, 0,1, \alpha_{R E} \chi_{2, l_{1}, l_{3}}, \alpha_{S R} \chi_{1, l_{1}, l_{2}}\right)$, where $\zeta_{2}\left(\eta, y_{l_{1}}, x_{l_{2}}\right)$ and $\bar{\xi}_{1}\left(y ; \kappa_{1}, \kappa_{2}, \kappa_{3}, a_{1}, a_{2}, b_{1}, b_{2}\right)$ given in Table III consist of $v$-th order modified Bessel functions of second kind (i.e., $\mathcal{K}_{v}(x)$ ), and $\mathcal{K}_{v}(x)$ can also be expressed as $\mathcal{K}_{v}(x)=\sqrt{\pi} e^{-x}(2 x)^{v} \Psi(v+0.5,1+2 v ; 2 x)$ [45, eq. (9.238)]. Realizing this in $\mathcal{Z}$ indicates that due to the presence of exponential functions, $\mathcal{Z}$ converges rapidly as $U_{1}, U_{2}$, and $U_{3}$ increase and only limited values are sufficient to get a satisfactory accuracy, as shown numerically in Section IV. Likewise, convergence of other terms can also be shown.

Remark 11: The $\mathcal{S}_{j}(\eta)$, for $j \in\{1,2,3,4\}$ in (14) consist infinite summations. We test the convergence as follows. For instance, consider a term from $\mathcal{S}_{1}(\eta)$ in (15), i.e., $\mathcal{M}_{u}=\frac{\left(\varpi_{k 1} \eta x_{l_{2}}\right)^{u} M^{u+2}}{u ! \alpha_{S E}^{2 u+3}} \frac{\alpha_{S R} \alpha_{R E}^{2} \Omega_{R P} z_{l_{3}} \phi_{k 1}(\eta)}{\sqrt{M}} \mathcal{N}_{2}\left(-\frac{3}{2}-u,-\frac{1}{2}-\right.$ $\left.u, 0,0, \frac{1}{2},-\frac{1}{2}, \frac{M \phi_{k 1}(\eta) \Omega_{R P} z_{l_{3}}}{x_{l_{2}}}, M\right)$, which is further expressed as $\mathcal{M}_{u}=G_{2,0: 0,2: 0,2}^{0,2: 2,0: 2,0}\left(\left.\left.\begin{array}{c}-\frac{3}{2}-u,-\frac{1}{2}-u \\ -\end{array}\right|_{0,0} ^{-}\right|_{\frac{1}{2},-\frac{1}{2}} ^{-} \mid \epsilon_{1}, \epsilon_{2}\right)$ $\times \frac{\left(M \varpi_{k 1} \eta\right)^{u} x_{l_{2}}^{u+1} \epsilon_{1} \epsilon_{2}}{u ! \sqrt{M} \alpha_{S E}^{2 u-1}}$, where $\epsilon_{1}=\frac{M \phi_{k 1}(\eta) z_{l_{3}} \Omega_{R P} \alpha_{R E}^{2}}{x_{l_{2}} \alpha_{S E}^{2}}$ and $\epsilon_{2}=\frac{M \alpha_{S R}^{2}}{\alpha_{S E}^{2}}$. Then, the series expansion of this term in (15) is given by $\sum_{u=0}^{\infty}(-1)^{u} \mathcal{M}_{u}$. It is well known fact that the extended generalized bi-variate Meijer- $G$ function, $\quad G_{p_{1}, q_{1}: p_{2}, q_{2}: p_{3}, q_{3}}^{m_{1}, n_{1}: n_{2}, m_{2}: n_{3}, m_{3}}\left(x,\left.\left.\left.y\right|_{b_{1}, \cdots, b_{q_{1}}} ^{a_{1}, \cdots, a_{p_{1}}}\right|_{d_{1}, \cdots, d_{q_{2}}} ^{c_{1}, \cdots, c_{p_{2}}}\right|_{f_{1}, \cdots, f_{q_{3}}} ^{e_{1}, \cdots, e_{3}}\right)$, can be defined in terms of double Mellin-Barnes type integrals, and converges if the following conditions satisfied [54], i.e., i) $p_{1}+p_{2}+q_{1}+q_{2}<2\left(n_{1}+n_{2}+m_{2}\right)$, ii) $p_{1}+p_{3}+q_{1}+q_{3}<2\left(n_{1}+n_{3}+m_{3}\right)$, iii) $|\arg (x)|<$ $\pi\left(n_{1}+n_{2}+m_{2}-\left(p_{1}+p_{2}+q_{1}+q_{2}\right) / 2\right)$, and iv) $|\arg (y)|<$ $\pi\left(n_{1}+n_{3}+m_{3}-\left(p_{1}+p_{3}+q_{1}+q_{3}\right) / 2\right)$. Thus, using ratio test, ${ }^{5}$ it can be shown that the series is convergent as $\lim _{u \rightarrow \infty} \frac{\mathcal{M}_{u+1}}{\mathcal{M}_{u}}$

\footnotetext{
${ }^{5}$ For a given infinite series $\sum_{u=0}^{\infty} f_{u}$, let $\mathcal{L}:=\lim _{u \rightarrow \infty}\left|\frac{f_{u+1}}{f_{u}}\right|$. As per [55], the series is convergent if $\mathcal{L}<1$, divergent if $\mathcal{L}>1$, and inclusive if $\mathcal{L}=1$.
}

$$
\begin{aligned}
& =\lim _{u \rightarrow \infty} \frac{M \varpi_{k 1} \eta x_{l_{2}}}{(u+1) \alpha_{S E}^{2}} \frac{\left.\left.\left.\begin{array}{c}
G_{2,0: 0,2: 0,2}^{0,2: 2,0: 2,0}\left({ }^{-\frac{3}{2}-u-1,-\frac{1}{2}-u-1}-\right. \\
-
\end{array}\right|_{0,0} ^{-}\right|_{\frac{1}{2},-\frac{1}{2}} ^{-} \mid \epsilon_{1}, \epsilon_{2}\right)}{G_{2,0: 0,2: 0,2}^{0,2: 2,0: 2,0}\left(\left.\left.{ }^{-\frac{3}{2}-u,-\frac{1}{2}-u}\right|_{0,0} ^{-}\right|_{\frac{1}{2},-\frac{1}{2}} ^{-} \mid \epsilon_{1}, \epsilon_{2}\right)} \\
& <\lim _{u \rightarrow \infty} \frac{M \varpi_{k 1} \eta x_{l_{2}}}{(u+1) \alpha_{S E}^{2}}=0 \text {. }
\end{aligned}
$$

We can infer that the infinite summations in Theorem 2 are convergent and thus making them practical and tractable to use. The convergence is numerically shown in Section IV.

\section{Effective Secrecy Diversity Order}

To extract some more meaningful information about the system's secrecy diversity order, this section investigates the effective secrecy diversity order of the considered system under mixed Rayleigh and double-Rayleigh fading, which to the best of authors' knowledge is yet to be studied in the literature. Therefore, next we investigate the effective secrecy diversity order by analyzing the asymptotic SOP behavior. Two scenarios ${ }^{6}$ pertaining to the wiretap channels, i.e., fixed wiretap average channel gains and varying wiretap average channel gains may arise, which are described as follows.

1) In the first scenario, the average channel gains related to the main links go to infinity, i.e., $\Omega_{S R} \rightarrow \infty, \Omega_{R D} \rightarrow$ $\infty$, and $\Omega_{S D} \rightarrow \infty$, and the average channel gains corresponding to wiretap channels i.e., $\Omega_{S E}$ and $\Omega_{R E}$ are fixed. The asymptotic analysis under this scenario will be helpful in understanding the system secrecy diversity order when the quality of the main channels is much better than the quality of the wiretap channels.

2) In the second scenario, both the main and wiretap channels go to infinity, i.e., $\Omega_{S R} \rightarrow \infty, \Omega_{R D} \rightarrow \infty$, $\Omega_{S D} \rightarrow \infty, \Omega_{S E} \rightarrow \infty$, and $\Omega_{R E} \rightarrow \infty$. This scenario is not considered in this paper, as it is obvious that in this scenario, perfect eavesdropping can be achieved, which results into a zero secrecy diversity order.

In what follows, we focus on the first scenario, when $\Omega_{i j} \rightarrow$ $\infty$, for $\{i j\} \in\{S R, R D, S D\}$, while $\Omega_{S E}$ and $\Omega_{R E}$ are fixed. Under this scenario, to evaluate the asymptotic SOP expression in the high- $\Omega_{i j}$ regime, we first need to obtain the asymptotic expression of the CDF of $\Theta$ conditioned on $X$ and $Z$ given in (24) under high- $\Omega_{i j}$ regime, which is given in Theorem 3.

Theorem 3: By simplifying (5), (6), (23), and (24) under high- $\Omega_{i j}$ regime, for $\{i j\} \in\{S R, R D, S D\}$, the asymptotic CDF of $\Theta$ conditioned on $X$ and $Z$ in the high- $\Omega_{i j}$ regime, keeping $\Omega_{S E}$ and $\Omega_{R E}$ as fixed, can be expressed as

$$
\begin{aligned}
& F_{\Theta}^{\mathrm{LB}}(\theta \mid X, Z) \underset{\Omega_{i j} \rightarrow \infty}{\simeq} \frac{4 \theta^{2} \Omega_{S E}^{4}}{\Omega_{R D} \Omega_{S D}} \frac{Z}{X}+\frac{4 \theta^{2} \Omega_{R E}^{4}}{\Omega_{R D} \Omega_{S D}} \frac{X}{Z} \\
& -\frac{\theta^{2} \Omega_{S E}^{5}}{\Omega_{R D} \Omega_{S D} \Omega_{R E}} \frac{Z^{\frac{3}{2}}}{X^{\frac{3}{2}}} G_{2,2}^{2,2}\left(\left.\frac{Z}{X} \frac{\Omega_{S E}^{2}}{\Omega_{R E}^{2}}\right|_{\frac{1}{2},-\frac{1}{2}} ^{-\frac{5}{2},-\frac{5}{2}}\right) \\
& -\frac{\theta^{2} \Omega_{R E}^{5}}{\Omega_{R D} \Omega_{S D} \Omega_{S E}} \frac{X^{\frac{3}{2}}}{Z^{\frac{3}{2}}} G_{2,2}^{2,2}\left(\left.\frac{X}{Z} \frac{\Omega_{R E}^{2}}{\Omega_{S E}^{2}}\right|_{\frac{1}{2},-\frac{1}{2}} ^{-\frac{5}{2},-\frac{5}{2}}\right) .
\end{aligned}
$$

Proof: See Appendix E for the detailed proof.

${ }^{6}$ Note that the realization of asymptotic SOP in the high $\rho=\frac{\mathcal{Q}}{N_{0}}$ regime also results into a zero secrecy diversity order, since $\rho$ at both $D$ and $E$ are increased simultaneously. 
Remark 12: Note that i) Theorem 3 does not involve any infinite summations and complicated mathematical functions, which makes it tractable for practical use, and ii) the desired asymptotic SOP can be obtained by averaging Theorem 3 over $X$ and $Z$, as given in Theorem 4, which provides various key insights into the system behavior as shown in Remarks 13-15.

Theorem 4: The asymptotic lower bound SOP expression at high- $\Omega_{i j}$ regime, for $\{i j\} \in\{S R, R D, S D\}$, can be given as

$$
\mathcal{P}_{\text {out, asy }}^{\text {sec, } \mathrm{LB}}(\eta)=\mathcal{G}_{c} \Omega^{-2},
$$

where $\Omega_{S R}=\Omega_{R D}=\Omega_{S D}=\Omega$ and $\mathcal{G}_{c}$ is the secrecy array gain given by

$$
\begin{aligned}
\mathcal{G}_{c} & =\frac{4 \eta^{2} \Omega_{S E}^{4} \Omega_{R P}}{\Omega_{S P}} \mathcal{T}\left(\Omega_{S P}\right)+\frac{4 \eta^{2} \Omega_{R E}^{4} \Omega_{S P}}{\Omega_{R P}} \mathcal{T}\left(\Omega_{R P}\right) \\
- & \frac{\eta^{2} \Omega_{S E}^{5} \Omega_{R P}^{\frac{3}{2}}}{\Omega_{R E} \Omega_{S P}^{\frac{3}{2}}} G_{3,3}^{3,3}\left(\left.\frac{\Omega_{S P}}{\Omega_{R P}} \frac{\Omega_{R E}^{2}}{\Omega_{S E}^{2}}\right|_{\frac{5}{2}, \frac{7}{2}, \frac{7}{2}} ^{\frac{3}{2}, \frac{1}{2}, \frac{3}{2}}\right) \\
- & \frac{\eta^{2} \Omega_{R E}^{5} \Omega_{S P}^{\frac{3}{2}}}{\Omega_{S E} \Omega_{R P}^{\frac{3}{2}}} G_{3,3}^{3,3}\left(\left.\frac{\Omega_{R P}}{\Omega_{S P}} \frac{\Omega_{S E}^{2}}{\Omega_{R E}^{2}}\right|_{\frac{5}{2}, \frac{7}{2}, \frac{7}{2}} ^{\frac{3}{2}, \frac{1}{2}, \frac{3}{2}}\right),
\end{aligned}
$$

with $\mathcal{T}(\beta)=\int_{0}^{\infty} \frac{1}{w} \mathrm{e}^{-\frac{w}{\beta}} d w$, which cannot be derived in closed form, however, it can be obtained numerically by the help of Mathematica or Matlab computation software packages.

Proof: See Appendix F for the detailed explanation.

Remark 13: It is evident from Theorem 4 that i) the system can achieve a secrecy diversity order of $\mathcal{G}_{\mathcal{D}}=2$, which is independent of the parameters related to $E$ and $P U$ receivers, ii) $\mathcal{P}_{\text {out, asy }}^{\text {sec, } \mathrm{LB}}(\eta)$ is directly proportional to $\mathcal{G}_{c}$, which implies that the asymptotic SOP is proportional to secrecy target rate, i.e., $\eta=2^{2 \mathcal{R}_{s}}$, and increases with the higher values of $\mathcal{R}_{s}$, as also reported in [15], [19], and [22], and iii) the utilization of $S \rightarrow D$ direct link gives significant benefits in system's secrecy diversity order, which is consistent with the findings reported in [10], [19], and [37].

Remark 14: Fig. 2 shows the effective secrecy diversity order behavior when $\Omega$ tends to infinity. It is observed from Fig. 2 that the secrecy diversity order convergence ${ }^{7}$ slows due to involved double-Rayleigh fading channels. The slow convergence behavior over cascaded fading channels is also proved in [56]. Mathematically, the secrecy diversity order can be defined as $\mathcal{G}_{\mathcal{D}}=-\lim _{\Omega \rightarrow \infty} \frac{\log \mathcal{P}_{\text {out, asy }}^{\text {sec }, ~}(\eta)}{\log \Omega}$. Now, using this and (18), we can compute the secrecy diversity order as

$$
\begin{aligned}
\mathcal{G}_{\mathcal{D}} & =\lim _{\Omega \rightarrow \infty}\left(2-\frac{\log \mathcal{G}_{c}}{\log \Omega}\right) \\
& =2 .
\end{aligned}
$$

We can infer that the term inside the limit in (20) converges to (21) very slowly as also shown in Fig. 2.

\footnotetext{
${ }^{7}$ Note that under path-loss channel modeling, the average power gains of all channels can be denoted as $\Omega_{\imath \jmath}=d_{\imath \jmath}^{-\nu}$, for $\{\imath \jmath\}=$ $\{S R, R D, S D, S E, R E, S P, R P\}$, with path-loss exponent $\nu=4$. It indicates that, $\Omega_{\imath \jmath} \rightarrow \infty$ in the above analysis correspond to $d_{\imath \jmath} \rightarrow 0$, which implies that the nodes $\imath$ and $\jmath$ are located close to each other. Whereas, $\Omega_{\imath \jmath} \rightarrow 0$ correspond to $d_{\imath \jmath} \rightarrow \infty$, which indicates that the node $\imath$ is located far away from the node $\jmath$. Therefore, the convergence behavior of the secrecy diversity order remains same under such a path-loss modeling, as the average power gains of the channels are directly related with the nodes position.
}

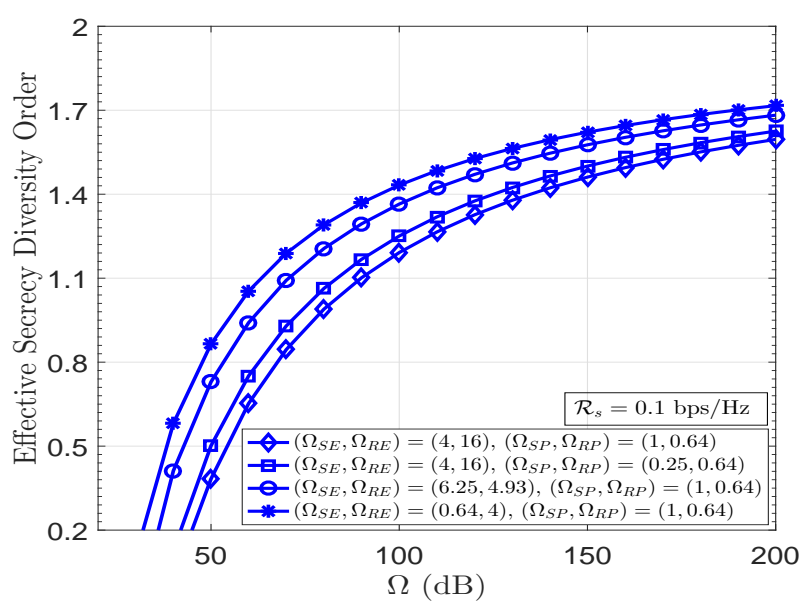

Fig. 2. Effective secrecy diversity order for the considered system.

Remark 15: From Fig. 2, it can be seen that the effective secrecy diversity order convergence further slows down when eavesdropper channel condition improves i.e., $\Omega_{S E}$ and $\Omega_{R E}$ increases, and vice versa. The same observation also holds when $\Omega_{S P}$ and $\Omega_{R P}$ increases. However, the secrecy diversity order of 2 can be achievable in all cases.

\section{NUMERICAL RESUltS AND Discussions}

In this section, we present numerical results to testify the accuracy of our derived analytical results via Monte-Carlo simulations (averaged over $10^{6}$ independent trials). For numerical investigations, we assume appropriate values of the orders of involved Gauss-Laguerre polynomial as $U_{1}=U_{2}=U_{3}=75$. The SOP expression in (14) consists infinite series, that converges after a few terms $(u=11)$ to get a precise result.

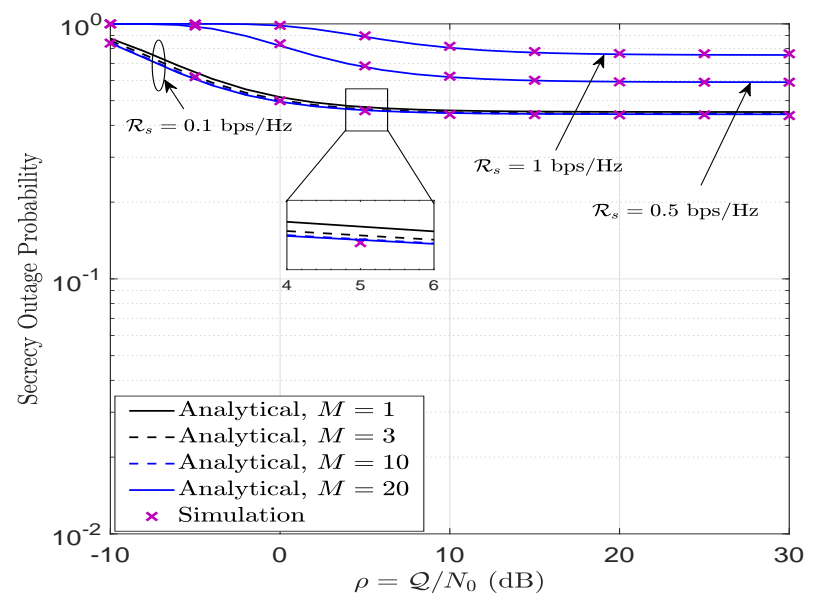

Fig. 3. SOP versus $\rho$ for different secrecy threshold $\mathcal{R}_{s}$.

Fig. 3 depicts the SOP performance of the considered system versus $\rho \triangleq \frac{Q}{N_{0}}$ for various values of $M$ and $\mathcal{R}_{s}$, when $\Omega_{\imath \jmath}=0 \mathrm{~dB}$ for $\{\imath \jmath\} \in\{S R, S E, S P, S D, R E, R P, R D\}$. From Fig. 3, we can see that the analytical results for SOP in (14) match well with the simulation results when $M \geq 10$ over 


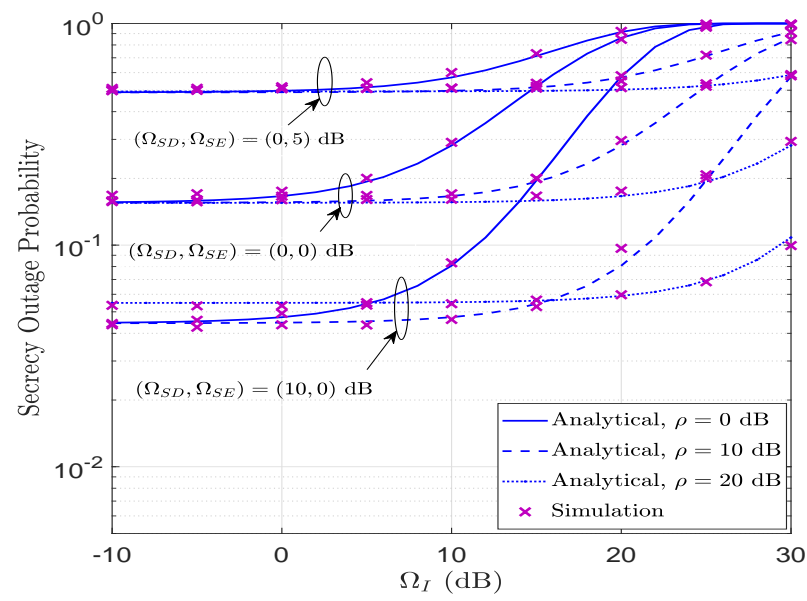

Fig. 4. Impact of primary user receiver on the SOP performance.

the entire SNR regime, and hence validating the correctness of our derived analytical SOP expression. It also verifies the accuracy of rectangular sub-regions approach [51] used to evaluate the SOP, as also shown in [37]. We can further observe that the SOP increases with the increase in $\mathcal{R}_{s}$, which is generally reported in [15], [19], and [22]. This is because of the reason that a higher power will be required to achieve the more stringent secrecy rate threshold constraints. Moreover, we can observe from Fig. 3 that the SOP curves exhibit secrecy floor phenomenon, irrespective of $\mathcal{R}_{s}$, as reported in [19]. The reason is that in this case even $E$ is able to extract the benefits of increased transmit SNR alongwith $D$.

In Fig. 4, we demonstrate the impact of $P U$ (assuming $\Omega_{S P}=\Omega_{R P}=\Omega_{I}$ ) on the SOP performance for various values of $\Omega_{S D}, \Omega_{S E}$, and $\rho$, when $\mathcal{R}_{s}=0.1 \mathrm{bps} / \mathrm{Hz}, M=10$, and $\Omega_{S R}=\Omega_{R D}=10 \mathrm{~dB}$. From Fig. 4, we can see that the SOP increases as $\Omega_{I}$ increases, irrespective of $\Omega_{S D}, \Omega_{S E}$, and $\rho$. This is owing to the reason that the powers $P_{S}$ and $P_{R}$ decrease with the increase in $\Omega_{I}$, which is in coherence with the observation drawn in [27], [41]. It is further seen that the SOP increases with the increase in $\Omega_{S E}$, since increasing $\Omega_{S E}$ can improve the channel quality of $S \rightarrow E$ direct link, and hence enhances $E$ 's ability to intercept the information. In addition, the SOP decreases with the increase in $\Omega_{S D}$ owing to a better legitimate $S \rightarrow D$ channel quality. The above two observations show that the direct links have significant impact on system's secrecy performance, and should not be neglected while analyzing PHY-security, which is also aligned with the findings reported in [19], [22], and [23]. Moreover, it is seen from Fig. 4 that the SOP performance shows an improvement with the increased $\rho$ in the medium-to-high $\Omega_{I}$ regime, which is consistent with the observation shown in [25].

Fig. 5 shows the SOP performance versus $\Omega$ (where $\Omega_{S R}=$ $\Omega_{R D}=\Omega_{S D}=\Omega$ ) for various values of $\Omega_{S E}$ and $\Omega_{R E}$, when $\mathcal{R}_{s}=0.5 \mathrm{bps} / \mathrm{Hz}, M=10, \Omega_{S P}=\Omega_{R P}=0 \mathrm{~dB}$, and $\rho=0$ dB. It can be seen from Fig. 5 that the SOP decreases with the improvement in $\Omega$, for all values of $\Omega_{S E}$ and $\Omega_{R E}$. This is due to the fact that the legitimate channel quality improves continuously with an increase in $\Omega$, while the quality of

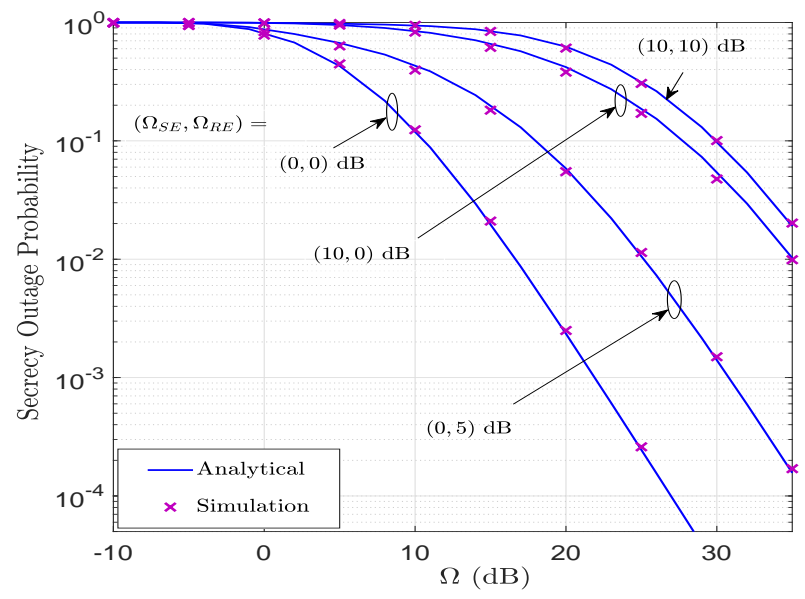

Fig. 5. SOP versus $\Omega$ for various values of $\Omega_{S E}$ and $\Omega_{R E}$.

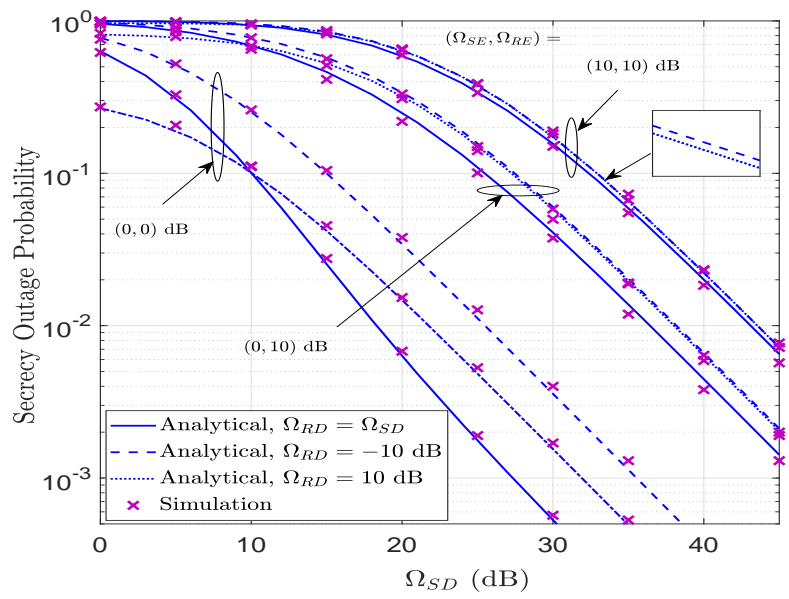

Fig. 6. Impact of $\Omega_{S D}$ on the SOP performance.

eavesdropper channels remains same due to the fixed $\Omega_{S E}$ and $\Omega_{R E}$ values. Such an observation with direct links is consistent with the findings reported in PHY-security literature for noncognitive framework [10] and [37] and cognitive scenario [19], [22]. We can also observe that the SOP performance degrades when $\Omega_{S E}$ or $\Omega_{R E}$ increases. For instance, when $\Omega=20 \mathrm{~dB}$, the SOP is 0.00234656 for $\left(\Omega_{S E}, \Omega_{R E}\right)=(0,0) \mathrm{dB}$, whereas it is 0.419791 and 0.0583698 for $\left(\Omega_{S E}, \Omega_{R E}\right)=(10,0) \mathrm{dB}$ and $\left(\Omega_{S E}, \Omega_{R E}\right)=(0,5) \mathrm{dB}$, respectively. Furthermore, the SOP performance degrades significantly if $\Omega_{S E}$ and $\Omega_{R E}$ increase jointly, i.e., $\Omega_{S E}=\Omega_{R E}=10 \mathrm{~dB}$.

Fig. 6 illustrates the performance of SOP versus average channel gain $\Omega_{S D}$ of the legitimate direct link for various fixed values of $\Omega_{S E}, \Omega_{R E}, \Omega_{R D}$, when $\mathcal{R}_{s}=0.5 \mathrm{bps} / \mathrm{Hz}, M=10$, $\Omega_{S R}=10 \mathrm{~dB}, \Omega_{S P}=\Omega_{R P}=0 \mathrm{~dB}$, and $\rho=10 \mathrm{~dB}$. From the plots, we can observe that the SOP performance improves as $\Omega_{S D}$ increases, irrespective of the other parameters involved. This is due to the fact that an increase in $\Omega_{S D}$ strengthens the capacity pertaining to the legitimate $S \rightarrow D$ channel, which is aligned with the findings shown in [22], [23]. Further, we can observe that the SOP decreases with the increase in $\Omega_{R D}$ 
because of the better quality of the corresponding legitimate $R \rightarrow D$ link quality. Thus, as the quality of either/both $S \rightarrow D$ link and $R \rightarrow D$ link improves, the SOP performance will further improve. Also, it is seen that the SOP performance degrades when $\Omega_{S E}$ and/or $\Omega_{R E}$ increases.

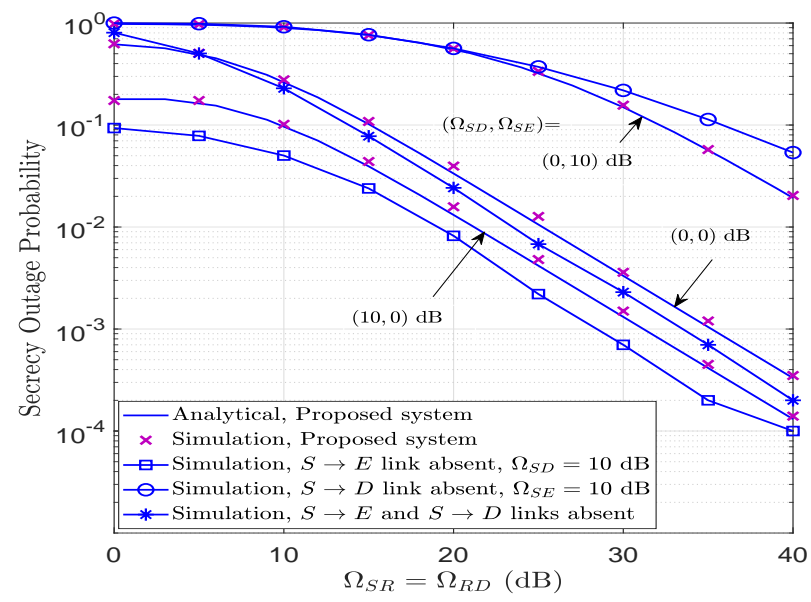

Fig. 7. SOP performance comparison from the direct links perspective.

Fig. 7 shows the performance of SOP versus average channel gains $\left(\Omega_{S R}=\Omega_{R D}\right)$ for the considered system, when $\mathcal{R}_{s}=0.5 \mathrm{bps} / \mathrm{Hz}, M=10, \Omega_{R E}=10 \mathrm{~dB}, \Omega_{S P}=\Omega_{R P}=0$ $\mathrm{dB}$, and $\rho=10 \mathrm{~dB}$. From Fig. 7, we can see that the SOP performance of the considered system improves as the quality of $S \rightarrow R$ and $R \rightarrow D$ channels improves, irrespective of $\Omega_{S D}$ and $\Omega_{S E}$. This gain in the secrecy performance is intuitive as an increase in $\Omega_{R D}$ brings a consistent gain to the capacity of legitimate relay channel $R \rightarrow D$, while $\Omega_{R E}$ is fixed and thus, limiting the capacity of the wiretap relay link $R \rightarrow E$. Moreover, we can see that SOP performance increases as $\Omega_{S D}$ increases, while an increase in $\Omega_{S E}$ can decrease the SOP performance. This is because of the reason that a higher $\Omega_{S D}$ $\left(\Omega_{S E}\right)$ corresponds to a better $S \rightarrow D(S \rightarrow E)$ channel capacity. Also, in Fig. 7, we consider four scenarios taken from our proposed system namely: 1) when both $S \rightarrow E$ and $S \rightarrow D$ are present (i.e., our considered system setup), 2) when $S \rightarrow E$ link is absent, 3) when $S \rightarrow D$ link is absent, and 4) when both $S \rightarrow E$ and $S \rightarrow D$ links are absent. We can observe that the SOP performance for scenario $2(S \rightarrow E$ link absent) outperforms the proposed system, whereas the proposed system (scenario 1) outperforms scenario $3(S \rightarrow D$ link absent). Such behavior is expected as the presence of $S \rightarrow E$ link strengthens the wiretap link capacity, while the presence of $S \rightarrow D$ link strengthens the main link capacity. Furthermore, the SOP performance of scenario $4(S \rightarrow E$ and $S \rightarrow D$ links absent) lies in between scenario 2 and scenario 3 . Moreover, the SOP performance of scenario 1 performs poorer than the scenario 4 when the average channel gain of $S \rightarrow E$ link is stronger than the average channel gain of $S \rightarrow D$ link, and outperforms when the quality of $S \rightarrow D$ link is superior than the quality of $S \rightarrow E$ link. Finally, we can conclude that the involvement of direct links have significant impact on system's SOP performance, as also reported in [19] and [22].

\section{Conclusions}

We investigated the secrecy performance of a secure underlay cooperative cognitive relay network, wherein a secondary source vehicle communicates with a fixed secondary infrastructure terminal via a direct link and with the help of a secondary AF relay vehicle in the presence of a secondary eavesdropper vehicle. We assumed that the eavesdropper could intercept the information from both the source and relay terminals, and also the secondary transmission occurs under the peak interference constraints to the primary user. Considering double-Rayleigh fading for $\mathrm{V} 2 \mathrm{~V}$ links and Rayleigh fading for V2I links, we deduced a novel tight lower bound expression for the SOP. We further presented an effective system's secrecy diversity order analysis, from which a secrecy diversity order of 2 can be achieved but with slow convergence rate due to the availability of double-Rayleigh fading channels. Furthermore, we verified our analytical results via simulations and showed the impacts of channel conditions and interference constraints on the SOP performance. Through our analysis, we also demonstrated that $S \rightarrow D$ and $S \rightarrow E$ direct links have considerable impacts on system's secrecy performance.

Future work will focus on the study of mitigating the eavesdropping effects by employing cooperative jamming or artificial noise in such networks. Also, the study of distributed beamforming and opportunistic relaying schemes for security enhancement in such networks is another topic of interest.

\section{APPENDIX A}

PROOF OF PROPOSITION 1

The conditioned CDF of $\widetilde{\Lambda}_{i}$, for $i \in\{D, E\}$, is given by

$$
\begin{aligned}
& F_{\widetilde{\Lambda}_{i}}(y \mid X, Z)=\operatorname{Pr}\left[\min \left(\Lambda_{S R}, \Lambda_{R i}\right)<y \mid X, Z\right] \\
& =F_{\Lambda_{S R}}(y \mid X)+F_{\Lambda_{R i}}(y \mid Z)-F_{\Lambda_{S R}}(y \mid X) F_{\Lambda_{R i}}(y \mid Z) .
\end{aligned}
$$

To obtain (22), we require the conditional CDFs $F_{\Lambda_{S R}}(y \mid X)$, $F_{\Lambda_{R D}}(y \mid Z)$, and $F_{\Lambda_{R E}}(y \mid Z)$. The CDF of $\Lambda_{S R} \triangleq \frac{\rho\left|h_{S R}\right|^{2}}{X}$ conditioned on $X$ can be given as $F_{\Lambda_{S R}}(y \mid X)=\operatorname{Pr}\left[\frac{\rho\left|h_{S R}\right|^{2}}{X}<\right.$ $y]=F_{\left|h_{S R}\right|^{2}}\left(\frac{y X}{\rho}\right)=1-2 \alpha_{S R} \sqrt{y X} \mathcal{K}_{1}\left(2 \alpha_{S R} \sqrt{y X}\right)$. Likewise, we can get the conditional CDFs of $\Lambda_{R D} \triangleq \frac{\rho\left|h_{R D}\right|^{2}}{Z}$ and $\Lambda_{R E} \triangleq \frac{\rho\left|h_{R E}\right|^{2}}{Z}$ as $F_{\Lambda_{R D}}(y \mid Z)=1-\mathrm{e}^{-\alpha_{R D} y Z}$, and $F_{\Lambda_{R E}}(y \mid Z)=1-2 \alpha_{R E} \sqrt{y Z} \mathcal{K}_{1}\left(2 \alpha_{R E} \sqrt{y Z}\right)$, respectively.

Finally, invoking the above derived conditional CDFs into (22), and after some simplifications, we can obtain $F_{\widetilde{\Lambda}_{D}}(y \mid X, Z)$ in (5) and $F_{\widetilde{\Lambda}_{E}}(y \mid X, Z)$ in (6).

\section{APPENDIX B}

\section{PROOF OF PROPOSITION 2}

Using the tight staircase approximation approach [51], we can express $F_{\digamma_{\imath}}^{\mathrm{LB}}(y \mid X, Z)$, for $\imath \in\{D, E\}$ in (4) as

$$
\begin{aligned}
F_{\digamma_{\imath}}^{\mathrm{LB}}(y \mid X, Z) & =\sum_{k=1}^{M} \int_{\tau_{k 1} y}^{\tau_{k} y} f_{\Lambda_{S \imath}}(w \mid X) d w \int_{0}^{y\left(1-\tau_{k 1}\right)} f_{\widetilde{\Lambda}_{\imath}}(v \mid X, Z) d v \\
& =\sum_{k=1}^{M}\left[\left(F_{\Lambda_{S \imath}}\left(\tau_{k} y \mid X\right)-F_{\Lambda_{S D}}\left(\tau_{k 1} y \mid X\right)\right)\right. \\
& \left.\times F_{\widetilde{\Lambda}_{\imath}}\left(\left(1-\tau_{k 1}\right) y \mid X, Z\right)\right] .
\end{aligned}
$$


From (23), the conditional CDF $F_{\digamma_{D}}^{\mathrm{LB}}(y \mid X, Z)$ can be obtained by invoking the CDFs $F_{\Lambda_{S D}}(y \mid X)$ and $F_{\widetilde{\Lambda}_{D}}(y \mid X, Z)$, and after some simplifications, as given in (7). Likewise, we can express $F_{\digamma E}^{\mathrm{LB}}(y \mid X, Z)$, as presented in (8).

\section{APPENDIX C}

\section{Proof of THEOREM 1}

The CDF of $\Theta$ conditioned on $X$ and $Z$ can be given by $F_{\Theta}^{\mathrm{LB}}(\theta \mid X, Z)=\int_{0}^{\infty} F_{\digamma_{D}}^{\mathrm{LB}}(\theta-1+\theta y \mid X, Z) f_{\digamma_{E}}^{\mathrm{LB}}(y \mid X, Z) d y$,

which can be further expressed by invoking (7) as

$$
\begin{aligned}
& F_{\Theta}^{\mathrm{LB}}(\theta \mid X, Z)=\sum_{k=1}^{M}\left\{\int_{0}^{\infty} \mathcal{C}_{1}(\theta, X) \mathrm{e}^{-\varpi_{k 1} \theta y X} f_{\digamma_{E}}^{\mathrm{LB}}(y \mid X, Z) d y\right. \\
& -\int_{0}^{\infty} \mathcal{C}_{2}(\theta, X) \mathrm{e}^{-\varpi_{k} \theta y X} f_{\digamma_{E}}^{\mathrm{LB}}(y \mid X, Z) d y \\
& -\int_{0}^{\infty} \mathrm{e}^{-\mu_{k 1}(y ; \theta, X, Z)} \zeta_{1}(y ; \theta, X) f_{\digamma_{E}}^{\mathrm{LB}}(y \mid X, Z) d y \\
& \left.+\int_{0}^{\infty} \mathrm{e}^{-\mu_{k, k 1}(y ; \theta, X, Z)} \zeta_{1}(y ; \theta, X) f_{\digamma_{E}}^{\mathrm{LB}}(y \mid X, Z) d y\right\}
\end{aligned}
$$

To evaluate (25), we first require the conditional PDF $f_{\digamma_{E}}^{\mathrm{LB}}(y \mid X, Z)$, which can be obtained by differentiating (8) with respect to $y$ by the use of $\frac{\partial^{n}}{\partial z^{n}}\left[z^{v / 2} \mathcal{K}_{v}(a \sqrt{z})\right]=$ $(-a / 2)^{n} z^{(v-n) / 2} \mathcal{K}_{v-n}(a \sqrt{z})$ [57, eq. (1.14.1.4)], as

$f_{\digamma_{E}}^{\mathrm{LB}}(y \mid X, Z)=\mathcal{U}_{1}(y \mid X, Z)-\mathcal{U}_{2}(y \mid X, Z)+\mathcal{U}_{3}(y \mid X, Z)+\sum_{j=2}^{M}$

$\times\left[\mathcal{U}_{4}(y \mid X, Z)+\mathcal{U}_{5}(y \mid X, Z)+\mathcal{U}_{6}(y \mid X, Z)+\mathcal{U}_{7}(y \mid X, Z)\right], \quad(26)$

where

$$
\begin{aligned}
& \mathcal{U}_{1}(y \mid X, Z)=\alpha_{S R} \alpha_{R E}^{2} \xi_{1}\left(y ; \frac{1}{2}, 1, \frac{1}{2}, 0,1, \alpha_{R E} \sqrt{Z}, \alpha_{S R} \sqrt{X}\right) \\
& +\alpha_{S R}^{2} \alpha_{R E} \xi_{1}\left(y ; 1, \frac{1}{2}, \frac{1}{2}, 1,0, \alpha_{R E} \sqrt{Z}, \alpha_{S R} \sqrt{X}\right), \\
& \mathcal{U}_{2}(y \mid X, Z)=\frac{\alpha_{S R} \alpha_{R E}^{2} \alpha_{S E}}{\sqrt{M}} \xi_{2}\left(y ; 1,1,1,1,0,1, \frac{\alpha_{S E} \sqrt{X}}{\sqrt{M}}\right. \\
& \left., \alpha_{R E} \sqrt{Z}, \alpha_{S R} \sqrt{X}\right)+\frac{\alpha_{S R}^{2} \alpha_{R E} \alpha_{S E}}{\sqrt{M}} \xi_{2}\left(y ; \frac{3}{2}, \frac{1}{2}, 1,1,1,0\right. \\
& \left., \frac{\alpha_{S E} \sqrt{X}}{\sqrt{M}}, \alpha_{R E} \sqrt{Z}, \alpha_{S R} \sqrt{X}\right)+\frac{\alpha_{S R} \alpha_{R E} \alpha_{S E}^{2}}{M} \\
& \times \xi_{2}\left(y ; \frac{3}{2}, \frac{1}{2}, 1,0,1,1, \frac{\alpha_{S E} \sqrt{X}}{\sqrt{M}}, \alpha_{R E} \sqrt{Z}, \alpha_{S R} \sqrt{X}\right), \\
& \mathcal{U}_{3}(y \mid X, Z)=\frac{1}{M} \alpha_{S E}^{2} \xi_{3}\left(y ; 1,0,0,0, \frac{\alpha_{S E} \sqrt{X}}{\sqrt{M}}\right), \\
& \mathcal{U}_{4}(y \mid X, Z)=-\alpha_{S E}^{2} \tau_{j 1} \xi_{3}\left(y ; 1,0,0,0, \alpha_{S E} \sqrt{\tau_{j 1} X}\right) \\
& +\alpha_{S E}^{2} \tau_{j} \xi_{3}\left(y ; 1,0,0,0, \alpha_{S E} \sqrt{\tau_{j} X}\right), \\
& \mathcal{U}_{5}(y \mid X, Z)=\alpha_{S R} \alpha_{R E} \alpha_{S E}^{2} \tau_{j 1}\left(1-\tau_{j 1}\right) \xi_{2}\left(y ; \frac{3}{2}, \frac{1}{2}, 1,0\right. \\
& \left., 1,1, \alpha_{S E} \sqrt{\tau_{j 1} X}, \alpha_{R E} \sqrt{\left(1-\tau_{j 1}\right) Z}, \alpha_{S R} \sqrt{\left(1-\tau_{j 1}\right) X}\right) \\
& -\alpha_{S R} \alpha_{R E} \alpha_{S E}^{2} \tau_{j}\left(1-\tau_{j 1}\right) \xi_{2}\left(y ; \frac{3}{2}, \frac{1}{2}, 1,0,1,1\right. \\
& \left., \alpha_{S E} \sqrt{\tau_{j} X}, \alpha_{R E} \sqrt{\left(1-\tau_{j 1}\right) Z}, \alpha_{S R} \sqrt{\left(1-\tau_{j 1}\right) X}\right),
\end{aligned}
$$

$\mathcal{U}_{6}(y \mid X, Z)=\alpha_{S R} \alpha_{R E}^{2} \alpha_{S E} \sqrt{\tau_{j 1}\left(1-\tau_{j 1}\right)^{3}} \xi_{2}(y ; 1,1,1,1$ $\left., 0,1, \alpha_{S E} \sqrt{\tau_{j 1} X}, \alpha_{R E} \sqrt{\left(1-\tau_{j 1}\right) Z}, \alpha_{S R} \sqrt{\left(1-\tau_{j 1}\right) X}\right)$ $-\alpha_{S R} \alpha_{R E}^{2} \alpha_{S E} \sqrt{\tau_{j}\left(1-\tau_{j 1}\right)^{3}} \xi_{2}(y ; 1,1,1,1,0,1$ $\left., \alpha_{S E} \sqrt{\tau_{j} X}, \alpha_{R E} \sqrt{\left(1-\tau_{j 1}\right) Z}, \alpha_{S R} \sqrt{\left(1-\tau_{j 1}\right) X}\right)$, $\mathcal{U}_{7}(y \mid X, Z)=\alpha_{S R}^{2} \alpha_{R E} \alpha_{S E} \sqrt{\tau_{j 1}\left(1-\tau_{j 1}\right)^{3}} \xi_{2}\left(y ; \frac{3}{2}, \frac{1}{2}, 1\right.$ $\left., 1,1,0, \alpha_{S E} \sqrt{\tau_{j 1} X}, \alpha_{R E} \sqrt{\left(1-\tau_{j 1}\right) Z}, \alpha_{S R} \sqrt{\left(1-\tau_{j 1}\right) X}\right)$ $-\alpha_{S R}^{2} \alpha_{R E} \alpha_{S E} \sqrt{\tau_{j}\left(1-\tau_{j 1}\right)^{3}} \xi_{2}\left(y ; \frac{3}{2}, \frac{1}{2}, 1,1,1,0\right.$ $\left., \alpha_{S E} \sqrt{\tau_{j} X}, \alpha_{R E} \sqrt{\left(1-\tau_{j 1}\right) Z}, \alpha_{S R} \sqrt{\left(1-\tau_{j 1}\right) X}\right)$.

The first integral (say $\mathcal{S}_{1}(\theta \mid X, Z)$ ) in (25) is given via (26) as

$$
\begin{aligned}
& \mathcal{S}_{1}(\theta \mid X, Z)=\mathcal{C}_{1}(\theta, X)\left[\int_{0}^{\infty} \mathrm{e}^{-\varpi_{k 1} \theta y X} \mathcal{U}_{1}(y \mid X, Z) d y\right. \\
& -\int_{0}^{\infty} \mathrm{e}^{-\varpi_{k 1} \theta y X} \mathcal{U}_{2}(y \mid X, Z) d y+\int_{0}^{\infty} \mathrm{e}^{-\varpi_{k 1} \theta y X} \mathcal{U}_{3}(y \mid X, Z) d y \\
& +\sum_{j=2}^{M} \int_{0}^{\infty} \mathrm{e}^{-\varpi_{k 1} \theta y X}\left(\mathcal{U}_{4}(y \mid X, Z)+\mathcal{U}_{5}(y \mid X, Z)\right. \\
& \left.\left.+\mathcal{U}_{6}(y \mid X, Z)+\mathcal{U}_{7}(y \mid X, Z)\right) d y\right]
\end{aligned}
$$

Using (27), the first integral (say $\mathcal{S}_{1}^{(a)}(\theta \mid X, Z)$ ) in (35) can be evaluated by applying $\mathrm{e}^{-x}=G_{0,1}^{1,0}\left(\left.x\right|_{0} ^{-}\right)$[46, eq. (01.03.26.0004.01)] and $\mathcal{K}_{v}(\sqrt{x})=\frac{1}{2} G_{0,2}^{2,0}\left(\frac{x}{4} \mid \frac{v}{2}, \frac{-v}{2}\right)$ [46, eq. (03.04.26.0009.01)], and via [46, eq. (07.34.21.0081.01)] as

$$
\begin{aligned}
& \mathcal{S}_{1}^{(a)}(\theta \mid X, Z)=\frac{Z}{X}\left(\frac{\alpha_{R E}}{\alpha_{S R}}\right)^{2} \mathcal{N}_{1}\left(\frac{\varpi_{k 1} \theta}{\alpha_{S R}^{2}}, \frac{Z}{X}\left(\frac{\alpha_{R E}}{\alpha_{S R}}\right)^{2}\right) \\
& +\frac{X}{Z}\left(\frac{\alpha_{S R}}{\alpha_{R E}}\right)^{2} \mathcal{N}_{1}\left(\frac{X \varpi_{k 1} \theta}{Z \alpha_{R E}^{2}}, \frac{X}{Z}\left(\frac{\alpha_{S R}}{\alpha_{R E}}\right)^{2}\right) .
\end{aligned}
$$

Invoking (28) into (35), the second integral (say $\left.\mathcal{S}_{1}^{(b)}(\theta \mid X, Z)\right)$ can be simplified using $\mathrm{e}^{-a x}=\sum_{n=0}^{\infty} \frac{(-a x)^{n}}{n !}$ [45, eq. (1.211.1)], [46, eq. (03.04.26.0009.01)], and [46, eq. $(07.34 .21 .0081 .01)]$ as

$$
\begin{aligned}
& \mathcal{S}_{1}^{(b)}(\theta \mid X, Z)=\sum_{u=0}^{\infty} \frac{(-1)^{u}\left(\varpi_{k 1} \theta\right)^{u}}{u !} \frac{M^{u+\frac{3}{2}}}{\sqrt{X} \alpha_{S E}^{2 u+3}} \alpha_{S R} \alpha_{R E} \\
& \times\left[\frac{Z \alpha_{R E}}{\sqrt{X}} \mathcal{N}_{2}\left(-\frac{3}{2}-u,-\frac{1}{2}-u, 0,0, \frac{1}{2},-\frac{1}{2}, \frac{M Z}{X}, M\right)+\sqrt{Z}\right. \\
& \times \alpha_{S R} \mathcal{N}_{2}\left(-\frac{3}{2}-u,-\frac{1}{2}-u, \frac{1}{2},-\frac{1}{2}, 0,0, \frac{M Z}{X}, M\right)+\frac{\sqrt{Z}}{\sqrt{M}} \\
& \left.\times \alpha_{S E} \mathcal{N}_{2}\left(-1-u,-1-u, \frac{1}{2},-\frac{1}{2}, \frac{1}{2},-\frac{1}{2}, \frac{M Z}{X}, M\right)\right] .
\end{aligned}
$$

Moreover, the third integral (say $\mathcal{S}_{1}^{(c)}(\theta \mid X, Z)$ ) of (35) can be evaluated by using (29) and [45, eq. (6.614.4)], as

$$
\mathcal{S}_{1}^{(c)}(\theta \mid X, Z)=\frac{\alpha_{S E}}{\sqrt{M \varpi_{k 1} \theta}} \mathrm{e}^{\frac{1}{2} \frac{\alpha_{S E}^{2}}{M \varpi_{k 1} \theta}} \mathcal{W}_{-\frac{1}{2}, 0}\left(\frac{\alpha_{S E}^{2}}{M \varpi_{k 1} \theta}\right) .
$$


Finally, the fourth integral (say $\mathcal{S}_{1}^{(d)}(\theta \mid X, Z)$ ) of (35) can be re-written as

$$
\begin{aligned}
& \mathcal{S}_{1}^{(d)}(\theta \mid X, Z)=\int_{0}^{\infty} \mathrm{e}^{-\varpi_{k 1} \theta y X} \mathcal{U}_{4}(y \mid X, Z) d y \\
& +\int_{0}^{\infty} \mathrm{e}^{-\varpi_{k 1} \theta y X} \mathcal{U}_{5}(y \mid X, Z) d y+\int_{0}^{\infty} \mathrm{e}^{-\varpi_{k 1} \theta y X} \mathcal{U}_{6}(y \mid X, Z) d y \\
& +\int_{0}^{\infty} \mathrm{e}^{-\varpi_{k 1} \theta y X} \mathcal{U}_{7}(y \mid X, Z) d y .
\end{aligned}
$$

By invoking $\mathcal{U}_{4}(y \mid X, Z)$ from (30) into the first integral (say $\mathcal{S}_{1}^{(d, 1)}(\theta \mid X, Z)$ ) of (39), and simplifying the required integrals via [45, eq. (6.614.4)], we can express $\mathcal{S}_{1}^{(d, 1)}(\theta \mid X, Z)$ as

$$
\begin{aligned}
\mathcal{S}_{1}^{(d, 1)}(\theta \mid X, Z) & =\frac{\alpha_{S E}}{\sqrt{\varpi_{k 1} \theta}}\left[-\sqrt{\tau_{j 1}} \mathrm{e}^{\frac{1}{2} \frac{\alpha_{S E}^{2} \tau_{j 1}}{\varpi_{k 1} \theta}} \mathcal{W}_{-\frac{1}{2}, 0}\left(\frac{\alpha_{S E}^{2} \tau_{j 1}}{\varpi_{k 1} \theta}\right)\right. \\
& \left.+\sqrt{\tau_{j}} \mathrm{e}^{\frac{1}{2} \frac{\alpha_{S E}^{2} \tau_{j}}{\varpi_{k 1} \theta}} \mathcal{W}_{-\frac{1}{2}, 0}\left(\frac{\alpha_{S E}^{2} \tau_{j}}{\varpi_{k 1} \theta}\right)\right] .
\end{aligned}
$$

By invoking (31) into the second integral (say $\mathcal{S}_{1}^{(d, 2)}(\theta \mid X, Z)$ ) of (39), and simplifying the resultant integrals via same method as used to obtain (37), we can get $\mathcal{S}_{1}^{(d, 2)}(\theta \mid X, Z)$ as

$$
\begin{aligned}
& \mathcal{S}_{1}^{(d, 2)}(\theta \mid X, Z)=\sum_{u=0}^{\infty} \frac{(-1)^{u}\left(\varpi_{k 1} \theta\right)^{u}}{u !} \frac{\alpha_{S R} \alpha_{R E} \sqrt{Z}}{\sqrt{X} \alpha_{S E}^{2(u+1)}}\left(1-\tau_{j 1}\right) \\
& \times\left[\frac { 1 } { \tau _ { j 1 } ^ { u + 1 } } \mathcal { N } _ { 2 } \left(-1-u,-1-u, \frac{1}{2},-\frac{1}{2}, \frac{1}{2},-\frac{1}{2}, \frac{\left(1-\tau_{j 1}\right) Z}{\tau_{j 1} X}\right.\right. \\
& \left., \frac{1-\tau_{j 1}}{\tau_{j 1}}\right)-\frac{1}{\tau_{j}^{u+1}} \mathcal{N}_{2}\left(-1-u,-1-u, \frac{1}{2},-\frac{1}{2}, \frac{1}{2},-\frac{1}{2}\right. \\
& \left.\left., \frac{\left(1-\tau_{j 1}\right) Z}{\tau_{j} X}, \frac{1-\tau_{j 1}}{\tau_{j}}\right)\right] .
\end{aligned}
$$

Likewise, we can simplify the third and fourth integral terms (say $\mathcal{S}_{1}^{(d, 3)}(\theta \mid X, Z)$ and $\mathcal{S}_{1}^{(d, 4)}(\theta \mid X, Z)$ ) of (39). Hence, invoking the solutions of $\mathcal{S}_{1}^{(d, 1)}(\theta), \mathcal{S}_{1}^{(d, 2)}(\theta \mid X, Z), \mathcal{S}_{1}^{(d, 3)}(\theta \mid X, Z)$, and $\mathcal{S}_{1}^{(d, 4)}(\theta \mid X, Z)$ into (39), we can get $\mathcal{S}_{1}^{(d)}(\theta \mid X, Z)$. Consequently, using $\mathcal{S}_{1}^{(a)}(\theta \mid X, Z), \mathcal{S}_{1}^{(b)}(\theta \mid X, Z), \mathcal{S}_{1}^{(c)}(\theta \mid X, Z)$, and $\mathcal{S}_{1}^{(d)}(\theta \mid X, Z)$, we can obtain $\mathcal{S}_{1}(\theta \mid X, Z)$, as given in (12).

Now, following the same steps as used to obtain (12), the second integral (say $\mathcal{S}_{2}(\theta \mid X, Z)$ ) in (25) can be evaluated by replacing $\mathcal{C}_{1}(\theta, X)$ by $\mathcal{C}_{2}(\theta, X)$ and $\varpi_{k 1}$ by $\varpi_{k}$ in (12).

Further, invoking (26) into the third integral (say $\mathcal{S}_{3}(\theta \mid X, Z)$ ) of (25), it is seen that the integrals associated with $\mathcal{S}_{3}(\theta \mid X, Z)$ are mainly of the following forms

$$
\begin{aligned}
& \int_{0}^{\infty} \mathrm{e}^{-c_{1} y} y^{q} \sqrt{c_{2}+c_{3} y} \mathcal{K}_{1}\left(c_{4} \sqrt{y\left(c_{2}+c_{3} y\right)}\right) \mathcal{K}_{1}\left(c_{4} \sqrt{y}\right) \\
& \times \mathcal{K}_{0}\left(c_{5} \sqrt{y}\right) d y \\
& \int_{0}^{\infty} \mathrm{e}^{-c_{1} y} y^{q} \sqrt{c_{2}+c_{3} y} \mathcal{K}_{1}\left(c_{4} \sqrt{y\left(c_{2}+c_{3} y\right)}\right) \mathcal{K}_{1}\left(c_{6} \sqrt{y}\right) \\
& \times \mathcal{K}_{0}\left(c_{7} \sqrt{y}\right) \mathcal{K}_{1}\left(c_{8} \sqrt{y}\right) d y \\
& \int_{0}^{\infty} \mathrm{e}^{-c_{1} y} y^{q} \sqrt{c_{2}+c_{3} y} \mathcal{K}_{1}\left(c_{4} \sqrt{y\left(c_{2}+c_{3} y\right)}\right) \mathcal{K}_{0}\left(c_{9} \sqrt{y}\right) d y
\end{aligned}
$$

whose exact solutions are intractable. Therefore, we first multiply and divide $\mathcal{S}_{3}(\theta \mid X, Z)$ of (25) with $e^{y}$, and then simplifying it by applying the Gauss-Laguerre method [52] to obtain (13). Also, the fourth integral (say $\mathcal{S}_{4}(\theta \mid X, Z)$ ) in (25) can be evaluated and expressed by replacing $\mu_{k}(y ; \theta, X, Z)$ with $\mu_{k, k 1}(y ; \theta, X, Z)$ in (13).

Lastly, invoking the solutions of $\mathcal{S}_{1}(\theta \mid X, Z), \mathcal{S}_{2}(\theta \mid X, Z)$, $\mathcal{S}_{3}(\theta \mid X, Z)$, and $\mathcal{S}_{4}(\theta \mid X, Z)$ into (25), we can get the tight lower bound expression of $F_{\Theta}^{\mathrm{LB}}(\theta \mid X, Z)$, as presented in (11).

\section{APPENDIX D \\ PROOF OF THEOREM 2}

By averaging (11) over $X$ and $Z$ alongwith the PDFs $f_{X}(x)=\frac{1}{\Omega_{S P}} \mathrm{e}^{-\frac{x}{\Omega_{S P}}}$ and $f_{Z}(z)=\frac{1}{\Omega_{R P}} \mathrm{e}^{-\frac{z}{\Omega_{R P}}}$, the lower bound of the SOP, $\mathcal{P}_{\text {sec }}^{\text {out, }} \mathrm{LB}(\eta)$, can be expressed as

$$
\begin{aligned}
& \mathcal{P}_{\mathrm{sec}}^{\text {out, } \mathrm{LB}(\eta)} \\
& =\sum_{k=1}^{M}[\underbrace{\frac{1}{\Omega_{S P} \Omega_{R P}} \int_{0}^{\infty} \int_{0}^{\infty} \mathcal{S}_{1}(\eta \mid x, z) \mathrm{e}^{-\frac{x}{\Omega_{S P}}} \mathrm{e}^{-\frac{z}{\Omega_{R P}}} d x d z}_{\triangleq \mathcal{S}_{1}(\eta)} \\
& -\underbrace{\frac{1}{\Omega_{S P} \Omega_{R P}} \int_{0}^{\infty} \int_{0}^{\infty} \mathcal{S}_{2}(\eta \mid x, z) \mathrm{e}^{-\frac{x}{\Omega_{S P}}} \mathrm{e}^{-\frac{z}{\Omega_{R P}}} d x d z}_{\triangleq \mathcal{S}_{2}(\eta)} \\
& -\underbrace{\frac{1}{\Omega_{S P} \Omega_{R P}} \int_{0}^{\infty} \int_{0}^{\infty} \mathcal{S}_{3}(\eta \mid x, z) \mathrm{e}^{-\frac{x}{\Omega_{S P}}} \mathrm{e}^{-\frac{z}{\Omega_{R P}}} d x d z}_{\triangleq \mathcal{S}_{3}(\eta)} \\
& +\underbrace{\frac{1}{\Omega_{S P} \Omega_{R P}} \int_{0}^{\infty} \int_{0}^{\infty} \mathcal{S}_{4}(\eta \mid x, z) \mathrm{e}^{-\frac{x}{\Omega_{S P}}} \mathrm{e}^{-\frac{z}{\Omega_{R P}}} d x d z}_{\triangleq \mathcal{S}_{4}(\eta)} .
\end{aligned}
$$

To simplify $\mathcal{S}_{1}(\eta)$ in (45), we first invoke $\mathcal{S}_{1}(\eta \mid X, Z)$ from (12) into (45), and then carried out the required integration, it is observed that the solution of most of the integrals in $\mathcal{S}_{1}(\eta)$ of (45) is intractable due to the involvement of $X$ and $Z$ in the complicated arguments of extended generalized bi-variate Meijer- $G$ functions. To simplify, we apply change of variables $\phi_{k 1}(\eta) x=x$ and $\frac{z}{\Omega_{R P}}=z$ with the Gauss-Laguerre method [52], and consequently, we can obtain $\mathcal{S}_{1}(\eta)$ as given in (15).

Now, using the steps as used to obtain (15), we can evaluate and represent $\mathcal{S}_{2}(\eta)$ in (45) by replacing $\varpi_{k 1}$ by $\varpi_{k}$ in (15).

Further, the solution of $\mathcal{S}_{3}(\eta)$ in (45) is intractable. To make analysis simple, we evaluate $\mathcal{S}_{3}(\eta)$ in (45) by first using the change of variables $\psi_{1, k 1}\left(\eta, y_{l_{1}}\right) x=x$ and $\psi_{2, k 1}\left(\eta, y_{l_{1}}\right) z=z$, and then using Gauss-Laguerre method [52], as shown in (16).

Moreover, $\mathcal{S}_{4}(\eta)$ in (45) can be simplified by following the same method as used to obtain (16), and can be represented by replacing $\psi_{1, k 1}\left(\eta, y_{l_{1}}\right)$ by $\psi_{1, k}\left(\eta, y_{l_{1}}\right)$ in (16).

\section{APPENDIX E}

\section{PROOF OF THEOREM 3}

By applying the approximations $x \mathcal{K}_{v}(x) \underset{x \rightarrow 0}{\approx} 1$ and $\mathrm{e}^{-x} \underset{x \rightarrow 0}{\approx}$ $1-x$ into (5), we can obtain $F_{\widetilde{\Lambda}_{D}}(y \mid Z) \simeq \frac{Z y}{\rho \Omega_{R D}}$. Further, invoking $F_{\Lambda_{S D}}(y \mid X)=1-\mathrm{e}^{-\frac{y X}{\rho \Omega S D}}$ with the use of $\mathrm{e}^{-x} \underset{x \rightarrow 0}{\approx}$ $1-x$ alongwith the approximation of $F_{\widetilde{\Lambda}}(y \mid Z)$, and keeping $M=1$ into (23), we can approximate $F_{\digamma_{D}}^{\mathrm{LB}}(y \mid X, Z)$ as

$$
F_{\digamma_{D}}^{\mathrm{LB}}(y \mid X, Z) \simeq \frac{Z X y^{2}}{\rho^{2} \Omega_{R D} \Omega_{S D}} .
$$


Moreover, using $x \mathcal{K}_{v}(x) \underset{x \rightarrow 0}{\approx} 1$ for the main links, we can express the conditioned $\mathrm{CDF} F_{\widetilde{\Lambda}_{E}}(y \mid Z)$ in (6) as

$$
F_{\widetilde{\Lambda}_{E}}(y \mid Z) \simeq 1-2 \sqrt{\frac{y Z}{\rho \Omega_{R E}^{2}}} \mathcal{K}_{1}\left(2 \sqrt{\frac{y Z}{\rho \Omega_{R E}^{2}}}\right) .
$$

Invoking (47) and $F_{\Lambda_{S E}}(y \mid X)=1-2 \sqrt{\frac{y X}{\rho \Omega_{S E}^{2}}} \mathcal{K}_{1}\left(2 \sqrt{\frac{y X}{\rho \Omega_{S E}^{2}}}\right)$ into (23) with $M=1$, and then differentiating with respect to $y$ via [57, eq. (1.14.1.4)], we can get $f_{\digamma_{E}}^{\mathrm{LB}}(y \mid X, Z)$ as

$$
\begin{aligned}
& f_{\digamma_{E}}^{\mathrm{LB}}(y \mid X, Z) \simeq \frac{2 X}{\rho \Omega_{S E}^{2}} \mathcal{K}_{0}\left(2 \sqrt{\frac{y X}{\rho \Omega_{S E}^{2}}}\right)\left[1-2 \sqrt{\frac{y Z}{\rho \Omega_{R E}^{2}}}\right. \\
& \left.\times \mathcal{K}_{1}\left(2 \sqrt{\frac{y Z}{\rho \Omega_{R E}^{2}}}\right)\right]+\frac{2 Z}{\rho \Omega_{R E}^{2}} \mathcal{K}_{0}\left(2 \sqrt{\frac{y Z}{\rho \Omega_{R E}^{2}}}\right) \\
& \times\left[1-2 \sqrt{\frac{y X}{\rho \Omega_{S E}^{2}}} \mathcal{K}_{1}\left(2 \sqrt{\frac{y X}{\rho \Omega_{S E}^{2}}}\right)\right] .
\end{aligned}
$$

Furthermore, using the approximation $\frac{1+x}{1+y} \simeq \frac{x}{y}$ [11], [50], we can re-express (24) at high- $\Omega_{i j}$ regime, for $\{i j\} \in$ $\{S R, R D, S D\}$, as

$$
F_{\Theta}^{\mathrm{LB}}(\theta \mid X, Z) \simeq \int_{0}^{\infty} F_{\digamma_{D}}^{\mathrm{LB}}(\theta y \mid X, Z) f_{\digamma_{E}}^{\mathrm{LB}}(y \mid X, Z) d y .
$$

By substituting $F_{\digamma_{D}}^{\mathrm{LB}}(\eta y \mid X, Z)$ from (46) and $f_{\digamma_{E}}^{\mathrm{LB}}(y \mid X, Z)$ from (48) into (49), we can express (49) as

$$
\begin{aligned}
& F_{\Theta}^{\mathrm{LB}}(\theta \mid X, Z) \simeq \frac{2 X^{2} Z \eta^{2}}{\rho^{3} \Omega_{R D} \Omega_{S D} \Omega_{S E}^{2}} \mathcal{I}_{1}+\frac{2 X Z^{2} \eta^{2}}{\rho^{3} \Omega_{R D} \Omega_{S D} \Omega_{R E}^{2}} \mathcal{I}_{2} \\
& -\frac{4 X^{2} Z^{\frac{3}{2}} \eta^{2}}{\rho^{\frac{7}{2}} \Omega_{R D} \Omega_{S D} \Omega_{R E} \Omega_{S E}^{2}} \mathcal{J}_{1}-\frac{4 X^{\frac{3}{2}} Z^{2} \eta^{2}}{\rho^{\frac{7}{2}} \Omega_{R D} \Omega_{S D} \Omega_{S E} \Omega_{R E}^{2}} \mathcal{J}_{2} \\
& \text { where } \quad \mathcal{I}_{\iota}=\int_{0}^{\infty} y^{2} \mathcal{K}_{0}\left(2 \sqrt{\frac{y \gamma}{\rho}}\right) d y \\
& \mathcal{J}_{\iota}=\int_{0}^{\infty} y^{\frac{5}{2}} \mathcal{K}_{0}\left(2 \sqrt{\frac{y \gamma}{\rho}}\right) \mathcal{K}_{1}\left(2 \sqrt{\frac{y \delta}{\rho}}\right) d y
\end{aligned}
$$

with $\gamma=\frac{X}{\Omega_{S E}^{2}}$ and $\delta=\frac{Z}{\Omega_{R E}^{2}}$ for $\iota=1$, and $\gamma=\frac{Z}{\Omega_{R E}^{2}}$ and $\delta=\frac{X}{\Omega_{S E}^{2}}$ for $\iota=2$. Then, applying the change of variables $\frac{y \gamma}{\rho}=t^{2}$ in (51), and simplifying via [45, eq. (6.561.16)], we can express $\mathcal{I}_{\iota}$, for $\iota \in\{1,2\}$, as $\mathcal{I}_{\iota}=\frac{2 \rho^{3}}{\gamma^{3}}$.

Moreover, applying [46, eq. (03.04.26.0009.01)] into (52), and simplifying using [46, 07.34.21.0011.01], we can obtain $\mathcal{J}_{\iota}=\frac{\rho^{\frac{7}{2}}}{4 \gamma^{7}} G_{2,2}^{2,2}\left(\left.\frac{\delta}{\gamma}\right|_{\frac{1}{2},-\frac{1}{2}} ^{-\frac{5}{2},-\frac{5}{2}}\right)$.

Now, invoking the results of $\mathcal{I}_{\iota}$ and $\mathcal{J}_{\iota}$, for $\iota \in\{1,2\}$, into (50), and after some involved manipulations, we can express the conditional $\mathrm{CDF} F_{\Theta}^{\mathrm{LB}}(\theta \mid X, Z)$ in the high- $\Omega_{i j}$ regime, for $\{i j\} \in\{S R, R D, S D\}$, as given in (17).

\section{APPENDIX F}

\section{PROOF OF THEOREM 4}

By unconditioned (17) over $X$ and $Z$, the asymptotic SOP, $\mathcal{P}_{\text {sec, asy }}^{\text {out } \mathrm{LB}}(\eta)$, at high- $\Omega_{i j}$, for $\{i j\} \in\{S R, R D, S D\}$, is given as

$$
\mathcal{P}_{\text {sec, asy }}^{\text {out, } \mathrm{LB}}(\eta)=\int_{0}^{\infty} \int_{0}^{\infty} F_{\Theta}^{\mathrm{LB}}(\eta \mid X, Z) f_{X}(x) f_{Z}(z) d x d z \text {. }
$$

Now, invoking $F_{\Theta}^{\mathrm{LB}}(\eta \mid X, Z)$ from (17) alongwith the PDFs of $X$ and $Z$ into (53), we can express $\mathcal{P}_{\text {sec, asy }}^{\text {out, }}(\eta)$ in (53) as

$$
\begin{aligned}
\mathcal{P}_{\text {sec, asy }}^{\text {out, } \mathrm{LB}}(\eta) & =\frac{1}{\Omega_{S P} \Omega_{R P}}\left[\frac{4 \eta^{2} \Omega_{S E}^{4}}{\Omega_{R D} \Omega_{S D}} \mathcal{T}_{1}+\frac{4 \eta^{2} \Omega_{R E}^{4}}{\Omega_{R D} \Omega_{S D}} \mathcal{T}_{2}\right. \\
& \left.-\frac{\eta^{2} \Omega_{S E}^{5}}{\Omega_{R D} \Omega_{S D} \Omega_{R E}} \mathcal{T}_{3}-\frac{\eta^{2} \Omega_{R E}^{5}}{\Omega_{R D} \Omega_{S D} \Omega_{S E}} \mathcal{T}_{4}\right]
\end{aligned}
$$

where

$$
\begin{aligned}
& \mathcal{T}_{1}=\int_{0}^{\infty} \int_{0}^{\infty} \frac{z}{x} \mathrm{e}^{-\frac{x}{\Omega_{S P}}} \mathrm{e}^{-\frac{z}{\Omega_{R P}}} d x d z \\
& \mathcal{T}_{2}=\int_{0}^{\infty} \int_{0}^{\infty} \frac{x}{z} \mathrm{e}^{-\frac{x}{\Omega_{S P}}} \mathrm{e}^{-\frac{z}{\Omega_{R P}}} d x d z \\
& \mathcal{T}_{3}=\int_{0}^{\infty} \int_{0}^{\infty} \frac{z^{\frac{3}{2}}}{x^{\frac{3}{2}}} G_{2,2}^{2,2}\left(\left.\frac{z}{x} \frac{\Omega_{S E}^{2}}{\Omega_{R E}^{2}}\right|_{\frac{1}{2},-\frac{1}{2}} ^{-\frac{5}{2},-\frac{5}{2}}\right) \mathrm{e}^{-\frac{x}{\Omega_{S P}}} \mathrm{e}^{-\frac{z}{\Omega_{R P}}} d x d z \\
& \mathcal{T}_{4}=\int_{0}^{\infty} \int_{0}^{\infty} \frac{x^{\frac{3}{2}}}{z^{\frac{3}{2}}} G_{2,2}^{2,2}\left(\left.\frac{x}{z} \frac{\Omega_{R E}^{2}}{\Omega_{S E}^{2}}\right|_{\frac{1}{2},-\frac{1}{2}} ^{-\frac{5}{2},-\frac{5}{2}}\right) \mathrm{e}^{-\frac{x}{\Omega_{S P}}} \mathrm{e}^{-\frac{z}{\Omega_{R P}}} d x d z
\end{aligned}
$$

Then, we can simplify $\mathcal{T}_{1}$ in (55) and $\mathcal{T}_{2}$ in (56) by applying the identity $\int_{0}^{\infty} w^{n} \mathrm{e}^{-\mu w} d w=n ! \mu^{-n-1}[45$, eq. (3.351.3)] as

$$
\begin{aligned}
& \mathcal{T}_{1}=\Omega_{R P}^{2} \int_{0}^{\infty} \frac{1}{x} \mathrm{e}^{-\frac{x}{\Omega_{S P}}} d x, \\
& \mathcal{T}_{2}=\Omega_{S P}^{2} \int_{0}^{\infty} \frac{1}{z} \mathrm{e}^{-\frac{z}{\Omega_{R P}}} d z .
\end{aligned}
$$

Furthermore, $\mathcal{T}_{3}$ in (57) can be simplified by applying the

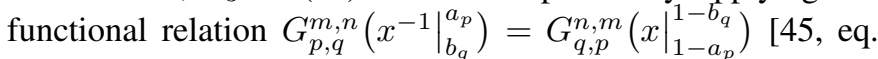
(9.31.2)] and by the use of [45, eq. (7.813.1)] as

$$
\mathcal{T}_{3}=\frac{\Omega_{R P}^{\frac{5}{2}}}{\Omega_{S P}^{\frac{1}{2}}} G_{3,3}^{3,3}\left(\left.\frac{\Omega_{S P}}{\Omega_{R P}} \frac{\Omega_{R E}^{2}}{\Omega_{S E}^{2}}\right|_{\frac{5}{2}, \frac{7}{2}, \frac{7}{2}} ^{\frac{3}{2}, \frac{1}{2}, \frac{3}{2}}\right) .
$$

Likewise, we can obtain $\mathcal{T}_{4}$ of (58) as

$$
\mathcal{T}_{4}=\frac{\Omega_{S P}^{\frac{5}{2}}}{\Omega_{R P}^{\frac{1}{2}}} G_{3,3}^{3,3}\left(\left.\frac{\Omega_{R P}}{\Omega_{S P}} \frac{\Omega_{S E}^{2}}{\Omega_{R E}^{2}}\right|_{\frac{5}{2}, \frac{7}{2}, \frac{7}{2}} ^{\frac{3}{2}, \frac{1}{2}, \frac{3}{2}}\right) .
$$

Then, substituting (59), (60), (61), and (62) into (54), and after some mathematical simplifications, we can get the asymptotic SOP expression in the high- $\Omega_{i j}$ regime, as presented in (18).

\section{REFERENCES}

[1] H. Zhou, W. Xu, J. Chen, and W. Weng, "Evolutionary V2X technologies toward the Internet of Vehicles: Challenges and opportunities," Proc. IEEE, vol. 108, no. 2, pp. 308-320, Feb. 2020

[2] K. B. Letaief and W. Zhang, "Cooperative communications for cognitive radio networks," Proc. IEEE, vol. 97, no. 5, pp. 878-893, May 2009.

[3] F. Mehmeti and T. Spyropoulos, "Performance analysis, comparison, and optimization of interweave and underlay spectrum access in cognitive radio networks," IEEE Trans. Veh. Tech., vol. 67, no. 8, pp. 7143-7157, Aug. 2018.

[4] KD. Singh, P. Rawat, and J-M. Bonnin, "Cognitive radio for vehicular ad-hoc networks (cr-vanets): approaches and challenges," EURASIP J. Wireless Commun. Netw., vol. 49, 2014.

[5] F. Salahdine and N. Kaabouch, "Security threats, detection, and countermeasures for physical layer in cognitive radio networks: A survey," Phy. Commun., vol. 39, Apr. 2020.

[6] Y. He, FR. Yu, Z. Wei, and V. Leung, "Trust management for secure cognitive radio vehicular ad-hoc networks," Ad Hoc Netw., vol. 86, pp. 154-165, Apr. 2019. 
[7] Y. Zou et al.,"Securing physical-layer communications for cognitive radio networks," IEEE Commun. Mag., vol. 53, no. 9, pp. 48-54, Sept. 2015.

[8] Z. Shu, Y. Qian, and S. Ci, "On physical layer security for cognitive radio networks," IEEE Netw., vol. 27, no. 3, pp. 28-33, June 2013.

[9] Y. Zou, J. Zhu, X. Wang, and L. Hanzo, "A survey on wireless security: Technical challenges, recent advances, and future trends," Proc. IEEE vol. 104, no. 9, pp. 1727-1765, Sept. 2016.

[10] L. Fan et al., "Exploiting direct links for physical layer security in multiuser multirelay networks," IEEE Trans. Wireless Commun., vol. 15, no. 6, pp. 3856-3867, June 2016.

[11] L. Fan et al., "Secure multiple amplify-and-forward relaying with cochannel interference," IEEE J. Sel. Topics Signal Process., vol. 10, no. 8, pp. 1494-1505, Dec. 2016.

[12] F. Ding et al., "Impact of relay's eavesdropping on untrusted amplifyand-forward networks over Nakagami- $m$ fading," IEEE Wireless Commun. Lett., vol. 7, no. 1, pp. 102-105, Feb. 2018.

[13] A. Omri and M. O. Hasna, "Average secrecy outage rate and average secrecy outage duration of wireless communication systems with diversity over Nakagami- $m$ fading channels," IEEE Trans. Wireless Commun., vol. 17, no. 6, pp. 3822-3833, June 2018.

[14] M. Yang et al., "Secure multiuser scheduling in downlink dual-hop regenerative relay networks over Nakagami- $m$ fading channels," IEEE Trans. Wireless Commun., vol. 12, no. 15, pp. 8009-8024, Dec. 2016.

[15] K. N. Le, "Secrecy and end-to-end analyses employing opportunistic relays under outdated channel state information and dual correlated Rayleigh fading," IEEE Trans. Veh. Technol., vol. 67, no. 11, pp. 1050410518, Nov. 2018

[16] K. N. Le and V. N. Q. Bao, "Secrecy under Rayleigh-dual correlated Rician fading employing opportunistic relays and an adaptive encoder," IEEE Trans. Veh. Technol., vol. 69, no. 5, pp. 5179-5192, May 2020.

[17] C. Ouyang et al., "Physical layer security over mixture Gamma distributed fading channels with discrete inputs: A unified and general analytical framework," IEEE Commun. Lett. (early access), DOI: 10.1109/LCOMM.2020.3025903, Sept. 2020.

[18] W. Saad, M. Shokair, and S. Ibraheem, "On the security of relay assisted cognitive radio networks in the presence of primary transceiver network," Wireless Pers. Commun., vol. 104, no. 3, pp. 949-977, Feb. 2019.

[19] P. Chakraborty and S. Prakriya, "Secrecy outage performance of a cooperative cognitive relay network," IEEE Commun. Lett., vol. 21, no. 2, pp. 326-329, Feb. 2017.

[20] H. Sakran et al., "Proposed relay selection scheme for physical layer security in cognitive radio networks," IET Commun., vol. 6, no. 16, pp. 2676-2687, Nov. 2012.

[21] M. Nguyen et al., "Secure cooperative half-duplex cognitive radio networks with $K$-th best relay selection," IEEE Access, vol. 5, pp. 66786687, May 2017.

[22] K. Chopra, R. Bose, and A. Joshi, "Secrecy performance of thresholdbased cognitive relay network with diversity combining," Journal of Commun. and Netw., vol. 20, no. 4, pp. 383-395, Aug. 2018.

[23] T. X. Quach, H. Tran, E. Uhlemann, and M. T. Truc, "Secrecy performance of cooperative cognitive radio networks under joint secrecy outage and primary user interference constraints," IEEE Access, vol. 8, pp. 18442-18455, Jan. 2020.

[24] X. Ding et al., "Relay selection for secrecy improvement in cognitive amplify-and-forward relay networks against multiple eavesdroppers," IET Commun., vol. 10, no. 15, pp. 2043-2053, Oct. 2016.

[25] W. Mou et al., "On the security of cooperative cognitive radio networks with distributed beamforming, " Eurasip J. Wireless Commun. Netw., vol. 2017, no. 1, pp. 144, 2017.

[26] R. Zhao, Y. Yuan, L. Fan, and Y.-C. He, "Secrecy performance analysis of cognitive decode-and-forward relay networks in Nakagami- $m$ fading channels," IEEE Trans. Commun., vol. 65, no. 2, pp. 549-563, Feb. 2017.

[27] H. Lei et al., "On secrecy outage of relay selection in underlay cognitive radio networks over Nakagami- $m$ fading channels," IEEE Trans. Cog. Commun. Netw., vol. 3, no. 4, pp. 614-627, Dec. 2017.

[28] A. Banerjee and S.P. Maity, "On residual energy maximization in cognitive relay networks with eavesdropping," IEEE Sys. J., vol. 13, no. 4, pp. 3836-3846, Dec. 2019.

[29] S. Jia, J. Zhang, H. Zhao, and R. Zhang, "Relay selection for improved security in cognitive relay networks with jamming," IEEE Wireless Commun. Lett., vol. 6, no. 5, pp. 662-665, Oct. 2017.

[30] P. Xie et al., "Cooperative jammer selection for secrecy improvement in cognitive Internet of Things," Sensors, vol. 18, pp. 4257-4272, 2018.

[31] M. Bouabdellah, F. El Bouanani, and M-S. Alouini, "A PHY layer security analysis of uplink cooperative jamming-based underlay CRNs with multi-eavesdroppers," IEEE Trans. Cog. Commun. Netw., vol. 6, no. 2, pp. 704-717, June 2020
[32] T. Zhang et al., "Secure transmission in cognitive MIMO relaying networks with outdated channel state information," IEEE Access, vol. 4 , pp. 8212-8224, Sep. 2016.

[33] Q. Yang, J. Ding, and A. Hu, "Secrecy outage performance analysis of DF cognitive relay network with co-channel interference," Wireless Pers. Commun., vol. 107, no. 1, pp. 549-564, July 2019.

[34] J. B. Andersen, "Statistical distributions in mobile communications using multiple scattering," in Proc. 27th URSI Gen. Assem., Aug. 2002.

[35] J. Salo, H. M. El-Sallabi, and P. Vainikainen, "Statistical analysis of the multiple scattering radio channel," IEEE Trans. Antennas Propag., vol. 54, no. 11, pp. 3114-3124, Nov. 2006.

[36] I. Z. Kovacs, "Radio channel charaterisation for private mobile radio systems-mobile-to-mobile radio link investigations," $\mathrm{PhD}$ thesis, Institut Elektroniske Systemer, Aalobrg Universitet, Aalborg, Denmark, 2002.

[37] A. Pandey and S. Yadav, "Physical layer security in cooperative AF relaying networks with direct links over mixed Rayleigh and doubleRayleigh fading channels," IEEE Trans. Veh. Tech., vol. 67, no. 11, pp. 10615-10630, Nov. 2018.

[38] A. Pandey and S. Yadav, "Performance evaluation of amplify-andforward relaying cooperative vehicular networks under physical layer security," Trans. Emerg. Telecommun. Tech., vol. 29, no. 12, e3534, Dec. 2018.

[39] J. Zhang and G. Pan, "Secrecy outage analysis with $K$ th best relay selection in dual-hop inter-vehicle communication systems," AEU - Int. J. Electron. Commun., vol. 71, pp. 139-144, Jan. 2017.

[40] A. Pandey and S. Yadav, "Physical layer security in cooperative amplify-and-forward relay networks over mixed Nakagami- $m$ and double Nakagami- $m$ fading channels: performance evaluation and optimization," IET Commun. vol. 14, no. 1, pp. 95-114, Jan. 2020.

[41] T. T. Duy et al., "Outage performance of cognitive cooperative networks with relay selection over double-Rayleigh fading channels," IET Commun. vol. 10, no. 1, pp. 57-64, Apr. 2016

[42] J. Lee, J. H. Lee, and S. Bahk, "Performance analysis for multi-hop cognitive radio networks over cascaded Rayleigh fading channels with imperfect channel state information," IEEE Trans. Veh. Technol., vol. 68, no. 10, pp. 10335-10339, Oct. 2019.

[43] P. M. Nam et al., "Security versus reliability study for multi-hop cognitive M2M networks with joint impact of interference constraint and hardware noises," in International Conference on Advanced Technologies for Communications (ATC), Ho Chi Minh City, 2018, pp. 259-264.

[44] S. Yadav and A. Pandey, "On the Secrecy Performance of Cooperative Cognitive Vehicular Relay Networks," IEEE International Conference on Advanced Networks and Telecommunications Systems (ANTS), BITS Pilani, Goa India, 16-19 Dec. 2019, pp. 1-6.

[45] I. S. Gradshteyn and I. M. Ryzhik, Tables of Integrals, Series, and Products, 6th ed. New York: Academic Press, 2000.

[46] The Wolfram Functions Site [Online]. Available: http://functions.wolfram.com/.

[47] C. Jiang, Y. Chen, and K. J. R. Liu, "Data-driven optimal throughput analysis for route selection in cognitive vehicular networks," IEEE J. Sel. Areas Commun., vol. 32, no. 11, pp. 2149-2162, Nov. 2014.

[48] M. Di Felice, R. Doost-Mohammady, K. R. Chowdhury, and L. Bononi, "Smart radios for smart vehicles: Cognitive vehicular networks," IEEE Veh. Technol. Mag., vol. 7, no. 2, pp. 26-33, June 2012.

[49] D. Niyato, E. Hossain, and P. Wang, "Optimal channel access management with QoS support for cognitive vehicular networks," IEEE Trans. Mobile Computing, vol. 10, no. 4, pp. 573-591, Apr. 2011.

[50] I. Krikidis, J. S. Thompson, and S. McLaughlin, "Relay selection for secure cooperative networks with jamming," IEEE Trans. Wireless Commun. vol. 8, No. 10, pp. 5003-5011, Oct. 2009.

[51] C. Zhang et al., "A unified approach for calculating the outage performance of two-way AF relaying over fading channels," IEEE Trans. Veh. Technol., vol. 64, no. 3, pp. 1218-1229, Mar. 2015.

[52] M. Abromowitz and I. A. Stegun, Handbook of Mathematical Functions with Formulas, Graphs, and Mathematical Tables, New York, NY, USA: Dover, 1970.

[53] I. S. Ansari et al., "A new formula for the BER of binary modulations with dual-branch selection over generalized-K composite fading channels," IEEE Trans. Commun., vol. 59, no. 10, pp. 2654-2658, Oct. 2011.

[54] B. L. Sharma and R. F. A. Abioduan, "Generating function for generalized function of two variables," in Proc. American Mathematical Society, vol. 46, no. 1, pp. 69-72, Oct. 1974.

[55] W. Rudin, Principles of Mathematical Analysis, 3rd edition. New York: McGraw-Hill, 1976.

[56] M. Uysal,"Diversity analysis of space-time coding in cascaded Rayleigh fading channels," IEEE Commun. Lett., vol. 10, no. 3, pp. 165-167, Mar. 2006. 
[57] Y. A. Brychkov, Handbook of Special Functions: Derivatives, Integrals, Series and Other Formulas, CRC Press, 2008.

[58] I. Krikidis, "Opportunistic relay selection for cooperative networks with secrecy constraints," IET Commun., vol. 4, no. 15, pp. 1787-1791, Oct. 2010.

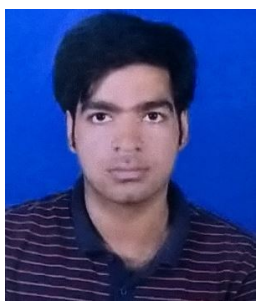

Anshul Pandey (S'17) received the B.Tech. degree in Electronics and Communication Engineering from PDPM-Indian Institute of Information Technology Design and Manufacturing, Jabalpur, Madhya Pradesh, India, in 2012, and the M.Tech. degree in Advance Networks from ABV-Indian Institute of Information Technology and Management, Gwalior, India, in 2016. He is currently pursuing the Ph.D. degree with the Department of Electronics and Communication Engineering, Indian Institute of Information Technology Allahabad, Prayagraj, India. His research interests include cooperative relaying for wireless vehicular networks, physical layer security, multiple-input-multiple-output communications, and signal processing.

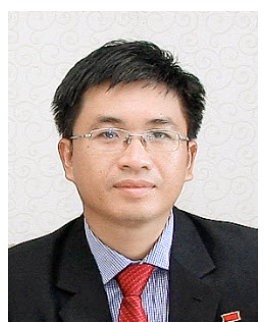

Dinh-Thuan Do (M'08-SM'09) received the B.S., M. Eng., and Ph.D. degrees from Vietnam National University (VNU-HCMC), in 2003, 2007, and 2013, respectively, all in communications engineering. $\mathrm{He}$ was a Visiting Ph.D. Student with the Communications Engineering Institute, National Tsing Hua University, Taiwan, from 2009 to 2010. Prior to joining Ton Duc Thang University, he was a Senior Engineer with VinaPhone Mobile Network, from 2003 to 2009. He was a founder of Wireless Communications Research Group funded by Ton Duc Thang University during period from 2016 to 2020 . He was a supervisor for numerous Ph.D. students in the Ph.D. joint program managed by Ton Duc Thang University and Technical University of Ostrava (VSB), Czech Republic. Dr. Thuan was a recipient of Golden Globe Award from Vietnam Ministry of Science and Technology in 2015 (Top 10 most excellent scientist nationwide). His publications include over 80 SCIE-indexed journal articles, over 100 SCOPUS-indexed journal articles and international conference papers. He is sole author in one textbook and one book chapter. His research interests include signal processing in wireless communications networks, NOMA, fullduplex transmission, and energy harvesting. He has served as a Guest Editor in Special Issue "UAV-enabled B5G/6G Networks: Emerging Trends and Challenges" PHYSICAL COMMUNICATION (Elsevier), 2021; a Lead Guest Editor in Special Issue "Power Domain Based Multiple Access Techniques in Sensor Networks" in INTERNATIONAL JOURNAL OF DISTRIBUTED SENSOR NETWORKS (IJDSN) in 2020; a Guest Editor in Special Issue "Massive sensors data fusion for health-care informatics", ANNALS OF TELECOMMUNICATIONS (Springer), 2020; a Lead Guest Editor in Special Issue "Recent Advances for 5G: Emerging Scheme of NOMA in Cognitive Radio and Satellite Communications", ELECTRONICS, 2019. He is currently serving as an Editor of COMPUTER COMMUNICATIONS (Elsevier), an Associate Editor of EURASIP JOURNAL ON WIRELESS COMMUNICATIONS AND NETWORKING (Springer), and an Editor of KSII TRANSACTIONS ON INTERNET AND INFORMATION SYSTEMS.

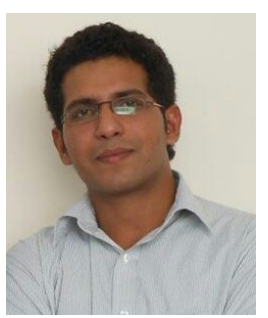

Suneel Yadav (S'13-M'16) has received the B.Tech. degree in Electronics and Communication Engineering from the Meerut Institute of Engineering and Technology, Meerut, India, in 2008, the M.Tech. degree in Digital Communications from the ABVIndian Institute of Information Technology and Management, Gwalior, India, in 2012, and the Ph.D. degree in the discipline of Electrical Engineering with the Indian Institute of Technology Indore, Indore, India, in 2016. He is currently working with the Department of Electronics and Communication Engineering, Indian Institute of Information and Technology Allahabad, Prayagraj, India, as an Assistant Professor. He is serving as a faculty incharge of Mobile and Wireless Networking Laboratory (MoWiNeT) at Indian Institute of Information and Technology Allahabad, Prayagraj, India. He has numerous publications in peer-reviewed journals and conferences. He also served as a TPC member, Session Chair, Program Co-Chairs, Reviewer, for various National and International Conferences. He is serving as a reviewer in a number of international journals including the IEEE TRANSACTIONS ON VEHICULAR TECHNOLOGY, the IEEE COMMUNICATIONS LETTERS, the IEEE TRANSACTIONS ON COMMUNICATIONS, the IEEE TRANSACTIONS ON INFORMATION FORENSICS AND SECURITY, the IEEE SYSTEMS JOURNAL, the IEEE ACCESS, the IEEE INTERNET OF THINGS JOURNAL, the IEEE TRANSACTIONS ON SIGNAL AND INFORMATION PROCESSING OVER NETWORKS, the PHYSICAL COMMUNICATION. His current research interests include wireless relaying techniques, cooperative communications, cognitive relaying networks, deviceto-device communications, reconfigurable intelligent surfaces, signal processing, physical layer security, and MIMO systems.

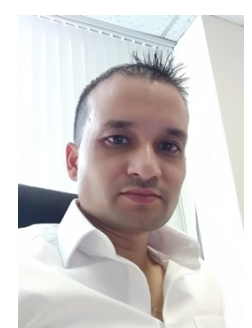

Rupak Kharel (M'09-SM'18) received the Ph.D. degree in secure communication systems from Northumbria University, U.K., in 2011. He is currently a Reader (Associate Professor) within the Department of Computing and Mathematics, Manchester Metropolitan University. His research interests include various use cases and the challenges of the IoT and cyber physical systems including Internet of Vehicles (IoV), cyber security, physical layer security, 5G and beyond systems. He is a Principal Investigator of multiple government and industry funded research projects. Rupak is a Senior member of the IEEE, member of the IET and a Fellow of the Higher Education Academy (FHEA), U.K. 